1 Out on a limb: bandicoot limb covariation suggests complex impacts of

2 development and adaptation on marsupial forelimb evolution

4 RUNNING HEADER: Limb integration in bandicoots

6 Kathleen Garland $^{\mathrm{a} *}$, Ariel Marcy ${ }^{\mathrm{a}}$, Emma Sherratt ${ }^{\mathrm{b}}$, Vera Weisbecker ${ }^{\mathrm{a} *}$

7

a School of Biological Sciences, University of Queensland, St. Lucia QLD 4072,

9 Australia

${ }^{\mathrm{b}}$ Department of Evolution, Ecology and Genetics, Research School of Biology,

11 The Australian National University, Canberra ACT, 2601 Australia

* Corresponding authors (School of Biological Sciences, University of

Queensland, St. Lucia QLD 4072, Australia; Phone +61 73365 7071; Fax: +61 7

Word count: 7,711 
Marsupials display far less forelimb diversity than placentals, possibly because of the laborious forelimb-powered climb to the pouch performed by most marsupial neonates. This is thought to result in stronger morphological integration (i.e. higher covariance) within the marsupial forelimb skeleton, and lower integration between marsupial fore- and hind limbs, compared to other mammals. Possible mechanisms for this constraint are a fundamental developmental change in marsupial limb patterning, or alternatively more immediate perinatal biomechanical and metabolic requirements. In the latter case, peramelid marsupials (bandicoots), which have neonates that climb very little, should show lower within-limb and higher between-limb integration, compared to other marsupials. We tested this in four peramelid species and the related bilby, using partial correlation analyses of between-landmark linear measurements of limb bones, and Procrustes-based two-block partial least-squares analysis (2B-PLS) of limb bone shapes using the same landmarks. We find extensive between-limb integration in partial correlation analyses of only bone lengths, consistent with a reduction of a short-term biomechanical/allocation constraint in peramelid forelimbs. However, partial correlations of bone proportions and 2B-PLS reveal extensive shape divergence between correlated bone pairs. This result contradicts expectations of developmental constraints or serial homology, instead suggesting a function-driven integration pattern. Comparing visualisations from cross-species principal components analysis and 2B-PLS, we tentatively identify selection for digging and halfbounding as the main driver of bandicoot limb integration patterning. This calls for further assessments of functional versus developmental limb integration in marsupials with a more strenuous neonatal climb to the pouch. 
The study of mammalian limb evolution has a long history (e.g. Cuvier 1800; Owen 1849) not least because mammalian diversity is reflected in the diversity of limbs, which are easily tractable proxies of functional adaptation (Polly 2007). The developmental similarities between the serially homologous fore- and hind limbs add to the appeal of limbs as an area where the relative impacts of intrinsic (genetic, structural or developmental) versus external (environmental) influences can be studied (Young and Hallgrímsson 2005; Polly 2007; Goswami et al. 2014). This is facilitated through the substantial variation of mammalian limb diversity. In particular, the largest mammalian clade - the placentals - displays a vast array of forelimb adaptations, while the smaller clade of marsupials has lower forelimb diversity (Sears 2004; Cooper and Steppan 2010; Kelly and Sears 2011; Weisbecker 2015). Marsupials also show fewer extreme deviations from their average forelimb shape, such as flippers, wings, or hooves (Lillegraven 1975; Polly 2007).

The restricted marsupial forelimb diversity is generally ascribed to their birth process. Nearly all of the highly altricial, minutely sized marsupial neonates climb from the urogenital sinus to the pouch using their well-developed forelimbs (Gemmell et al. 2002). This early requirement seems to fix marsupial forelimbs into a shape adapted for climbing (Lillegraven 1975; Kirsch 1977; Sears 2004; Sánchez-Villagra et al. 2008). By contrast, the much less-developed hind limbs are inactive during the climb, possibly allowing the later development of 'non-climbing' morphological adaptations (Sears 2004; Cooper and Steppan 2010; Keyte and Smith 2010; Bennett and Goswami 2011; Kelly and Sears 2011; Sears et al. 2012).

Aside from the morphological differences at birth, the developmental timing, modularity, and early gene expression differ between the front and back of the developing 
marsupial skeleton (Bininda-Emonds et al. 2007; Sánchez-Villagra et al. 2008; Goswami et al.

68

2009; Sears et al. 2012; Chew et al. 2014). In contrast, placental and monotreme fore- and hind limbs appear to develop with similar timing, like other vertebrates (Sánchez-Villagra 2002; Bininda-Emonds et al. 2007; Weisbecker et al. 2008; Sears 2009; Weisbecker 2011). Marsupial limb development is therefore most likely a derived trait, atypical for vertebrates (Weisbecker et al. 2008; Weisbecker 2011), contrary to earlier impressions (Lillegraven 1975; Hughes and Hall 1993; see also Weisbecker 2015).

Previous studies have used limb integration to assess whether the developmental constraint on marsupial limbs impacts on limb variation in adult populations (Bennett and Goswami 2011; Kelly and Sears 2011). Morphological integration between a pair of traits is high when their shape co-varies (Cheverud 1982; Klingenberg 2014). In particular, serially homologous traits (such as fore- and hind limbs) share a common developmental program thought to result in similar levels of shape co-variation (Young and Hallgrímsson 2005; but see Diogo and Ziermann 2015; Sears et al. 2015). Developmental changes, such as those suspected for marsupial forelimbs, are expected to break such patterns of covariation, offering a possible explanation for the apparent lack of evolvability in the marsupial forelimb (Fig. 1b; Bennett and Goswami 2011; Kelly and Sears 2011; Goswami et al. 2014). Several studies of intraspecific integration of marsupial limbs (summarized in Fig. 2; Bennett and Goswami 2011; Kelly and Sears 2011; Goswami et al. 2014) have supported this idea: Marsupials display greater between-limb and lower within-limb integration than placentals. Monotremes, particularly echidnas, also resemble placentals more than marsupials through greater integration between serially homologous fore- and hind limb bones (Bennett and Goswami 2011; Kelly and Sears 2011). 
Although studies of marsupial integration are consistent with a developmental constraint that restricts forelimb evolution and decouples it from that of the hind limbs (Fig. 1b; Sears 2004; Bennett and Goswami 2011; Kelly and Sears 2011), the mechanism for this constraint is not well understood. As noted above, substantial developmental disparity between marsupial fore- and hind limbs during pre-natal development suggests that forelimb constraints may arise during early, genetically-mediated changes to forelimb specification and development (Bininda-Emonds et al. 2007; Keyte and Smith 2010; Keyte and Smith 2012). On the other hand, the intense muscle strain on the developing marsupial shoulder girdle may present a more immediate biomechanical, rather than fundamental genetic, constraint on marsupial forelimb shape (Sears 2004; Weisbecker et al. 2008). This biomechanical process may be associated with a short-term shift in within-body resource allocation, leading to the disproportionate development of marsupial forelimbs (Keyte and Smith 2010; Keyte and Smith 2012). This would be consistent with recent suggestions that functional selection - for climbing into the pouch, in marsupials - has extensive, and possibly dominant, effects over the underlying vertebrate limb integration pattern reflecting serial homology (res minus Diogo papers); some workers even suggest that developmental integration due to serial homology does not exist (Fig. 1d; Wagner and Altenberg 1996; Diogo et al. 2013; Diogo and Molnar 2014; Klingenberg 2014; Linde-Medina and Diogo 2014; Martín-Serra et al. 2014; Diogo and Ziermann 2015). Such a biomechanical constraint would be more quickly reversed than a genetic constraint on marsupials as a whole (Weisbecker et al. 2008). In this case, an ancestral, presumably more placental- and possibly monotremelike pattern of higher between-limb and lower within-limb integration would be expected to re-emerge if a marsupial clade lost the functional constraints of the climb to the pouch. 
114 the impact of the climb to the pouch on marsupial limb evolution (Sears 2004; Weisbecker et al. 2008; Bennett and Goswami 2011). Bandicoot neonates slither down into the pouch with little forelimb action (Gemmell, Veitch, and Nelson 2002)., suggesting that a reduced developmental constraint on bandicoot forelimbs (Sears 2004; Cooper and Steppan 2010; Bennett and Goswami 2011), should increase the potential of bandicoot forelimbs to diversify. There is some evidence for this: bandicoots have placental-like fore- and hind limb ossification patterns (Sears 2004; Weisbecker et al. 2008), divergent scapular morphology from other marsupials (Sears 2004), and the most derived limbs among marsupials aside from the marsupial mole (Cooper and Steppan 2010), including an ossified patella and lack of a clavicle (Szalay 1994; Reese et al. 2001; Warburton et al. 2013). Peramelemorphs are also the only marsupial clade with a representative that has evolved a hoof-like forelimb (the recently extinct pig-footed bandicoot; Strahan 2004). whether integration patterns in limb long bones of four peramelid species support the hypothesis of a reduced developmental constraint on the forelimb compared to other marsupials. If this is the case, we expect to see lower within-limb and higher between-limb integration, as well as increased between-limb integration of limb bones that are functionally coupled (Fig. 1). We also compare peramelids with their sister species, the bilby,

132 whose neonates complete an extensive climb (Tait 2001). Lastly, we provide a preliminary assessment of the extent to which functional integration may contribute to bandicoot limb integration patterning. 


\section{Data acquisition}

138 Four adult and sub adult peramelid species were used for this study: the southern brown bandicoot, Isoodon obesulus ( $n=56)$, the eastern barred bandicoot, Perameles gunnii $(n=29)$,

140 the long-nosed bandicoot, Perameles nasuta $(n=12)$, the northern brown bandicoot, Isoodon macrourus $(n=8)$ and the sister species of peramelids (Mitchell et al. 2014), the greater bilby Macrotis lagotis ( $n=10$ ); for accession numbers, see Table S1. Photos of disarticulated limb bones (humerus, radius, metacarpal IV, femur, tibia, metatarsal IV) for each of the specimens were taken according to protocols in Bennett and Goswami (2011), and landmarked in tpsDIG2 v. 2.17 (Rohlf 2013). Older subadults with emerging $4^{\text {th }}$ molars and distinctive sagittal crests (Kingsmill 1962; Flores et al. 2013) were included, but assessed in detail for differences (described below). Each landmark represented a functionally homologous point of muscle insertion (Fig. S1; Table S2).

\section{Linear distances datasets}

A summary of the measurement acquisition and analysis workflows are in Fig. 3.

Linear distance measurements between all landmarks within each bone, were calculated using PAST (ver.2.17b; Hammer and Harper 2006) and regressed against the geometric mean of a single bone type within each species (Jungers et al. 1995) to remove isometric variation. Conventional 'length-only' (Young and Hallgrímsson 2005; Bennett and Goswami 2011; Kelly and Sears 2011) as well as 'multiple-distance' datasets (Fig. S1) were generated

157 (supplementary code and data). Multiple-distance datasets were derived from the averages of distances between each landmark with neighbouring landmarks for each bone. This 
compromises between length-only data - capturing length but not proportion (Weisbecker and Warton 2006) - and all-distance datasets with redundant information (Young and Hallgrímsson 2005). Random skewers analysis (Fig. 3.1.2) on variance/covariance (V/CV) matrices of all bone measurements for each species was conducted as a widely used measure of sample reliability (Cheverud and Marroig 2007).

\section{Procrustes residuals datasets}

Landmark coordinates (supplementary code and data) of each bone type of each species were aligned using a generalised Procrustes superimposition (GPA;Rohlf and Slice 1990), implemented in the R package geomorph v.3.0.1 (Adams and Otárola-Castillo 2013) in R 3.2.3 (R Development Core Team 2016; see supplementary code). A separate GPA was done for each bone and each species. The resulting Procrustes residual datasets characterise shape by preserving the geometry of each bone.

\section{Allometry and population sub-structure}

To assess whether allometry, location, sex, age, or side of the body contributed to the variation in bone shape, we ran Procrustes ANOVAs (Goodall 1991) with 1000 permutations/analysis as implemented in the R package geomorph both for shape and the multivariate distance matrix (see supplementary code), according to the formula

$$
\text { Shape or multiple-distance matrix Size+Sex+Location+Age+Side, }
$$

omitting specimens for which information was lacking (14 out of 56 in I. obesulus, 6 out of 29 in $P$. nasuta). We also ran an analysis of size differences and interactions between 
shape/linear distances, size (geometric mean/centroid size), and age structure (adults vs. subadults) to exclude issues of different allometric structure in these two groups. This also increased the dataset (48 out of 56 in I. obesulus, all 29 P. nasuta).

\section{Partial correlation and PC biplot analysis}

We conducted partial correlation analyses of the length-only and multiple-distance datasets, implemented in the R package corpcor (v. 1.4.3; Schaefer et al. 2013; see supplementary code). Edge exclusion deviance (EDD; Magwene 2001) was used to assess significance at values larger than $3.85(P<0.05, d f=1$, Chi-squared distribution; Magwene 2001; Bennett and Goswami 2011). Partial correlation results on length-only data were compared to published results from previous studies (Figs. 3-5, S2); (Young and Hallgrímsson 2005; Bennett and Goswami 2011; Kelly and Sears 2011)

Using the R package vegan (Oksanen et al. 2007), we also conducted principal component (PC) biplot analysis on our linear measurement datasets (supplementary code). These produce loading vectors whose angles represent positive or negative covariance between variables (bones in our case; Greenacre 2010). The null hypothesis of no difference between the PC biplot axes and the original limb dataset was assessed by comparing the observed squared correlation coefficient between randomly permuted datasets (1000 permutations). 


\section{Procrustes-shape 2B-PLS and PCA}

The association between bones for each species was assessed using a two-block partial least-squares analysis (2B-PLS, Rohlf and Corti 2000), implemented in geomorph. Statistical significance of the 2B-PLS-correlation was assessed by comparing the observed correlation to correlations generated from 10,000 random permutations of the original shape configurations. Shape changes associated with the first PLS axes of each bone pair were visualized as changes from the mean shape to the minima and maxima of the axis using thin-plate spline warp grids.

To compare among-species differences against within-species integration patterns, we performed a principal component analysis (PCA) for each bone, visualizing shape changes with thin-plate spline grids.

\section{Rarefaction analyses}

Sample size is likely to influence the results of the partial correlation analyses of linear distances and 2B-PLS analyses of Procrustes residuals. Therefore, we used our best-sampled species (I. obesulus and $P$. gunnii) to test the impact of sample size on the partial correlation Edge Exclusion Deviance (EED) and 2B-PLS correlation coefficient values through rarefaction. This involved taking a random sample of specimens at a range of sample sizes and computing the partial or correlation correlations with associated significances (EED/r-PLS), repeated 1000 times for each sample size. Rarefactions were conducted in steps of ten: $\mathrm{n}=10-50$ for $I$. obesulus (total $n=56$ ) and $n=10-20$ for $P$. gunnii (total $n=29$ ). For both partial correlation and 2B-PLS, three bone pairs per species with high, medium, and low-strength associations were chosen based on the results in the original full-sample analysis. 


\section{RESULTS}

Repeatability, allometry and population sub-structuring

Repeatability of V/CV matrices was high and at or above 90 for all species, even at low sample sizes (Table S3). The population sub-structuring analyses (Table S4) revealed little impact of sex on either linear distances of Procrustes shapes in either I. obesulus, with the only two exceptions in the linear distance data of the metacarpal and metatarsal of $P$. gunnii. Location had a significant effect on the variation in linear and Procrustes shape of only the tibiae and metatarsals of both I. obesulus and P. gunnii. Significant differences in the Procrustes residuals (but not linear measurements) were detected between the left and right radii and tibiae in $I$. obesulus and in the humerus of $P$. nasuta. Separate assessment of just size and age (Table S5) revealed significant allometry in both species but only a single, very low-level $(p=0.038)$ significant interaction between size and age in the metatarsals $P$. gunnii, which leads us to conclude that the incorporation of subadults in our dataset is not problematic.

Length-only partial correlations (Fig. 4; Table S6) and comparisons with published data from other mammals (Fig. 2) Species with small sample sizes tended to have higher partial correlation values than better-sampled species, which leads us to focus here on the two better-sampled species $I$. obesulus and P. gunnii. Generally, the placentals, monotremes (Fig. 2) and peramelids (Fig. 4, Table S6) displayed a higher number of significant partial correlations than other marsupials. I. obesulus, P. gunnii, and placentals also exhibited more instances of significant 
partial correlations between limbs than other marsupials. Isoodon obesulus was the only marsupial to resemble placentals and monotremes in having a significant positive lengthonly partial correlation between the humerus and femur.

Multiple-distance partial correlations (Fig. 4, Table S7) and biplots (Fig. 6, Table S9)

Nearly all significant multiple-distance based partial correlations (Fig. 5) in the peramelids are negative, in contrast to the length-only partial correlations which are mostly positive (Fig 4, Table S6). As seen in the length-only partial correlations, the multipledistance results for peramelids have more significant partial correlations between limbs than within limbs. However, the two best-sampled species (I. obesulus and $P$. gunnii) differ substantially in the pairs of bones showing significant partial correlation, hinting at considerable between-species variability of partial correlation patterning within peramelids.

The only significant partial correlations the two species share are negative ones between humerus/radius, humerus/tibia, and femur/metacarpal, and marginal positive ones between metacarpal/metatarsal. The bilby was the only peramelemorph to display a positive correlation between bone pairs other than the metacarpal and metatarsal (humerus/radius and radius/metatarsal). PC biplots (Fig. 6, Table S8) confirm these results: for example, multiple-distance data of metacarpals and metatarsals of $I$. obesulus and $P$. gunnii have high positive partial correlation values (Fig. 5), as well as long biplot vector lengths, and similar vector angles (Fig. 6). In contrast, the humerus and tibia of both species other at wide angles (Fig. 6). 
Two-block partial least-squares analysis (Figs. 7-9, Table S9)

As with partial correlation analyses, species with small sample sizes ( $M$. lagotis, $P$. nasuta, and I. macrourus) tended to have higher r-PLS-coefficients than the better-sampled I. obesulus and P. gunnii, so that again our interpretations are largely based on the latter two. I. obesulus and P. gunnii 2B-PLS results (Fig. 7 and Table S9) resemble the multipledistance partial correlation patterns, although overall more significant associations between bone pairs are found in 2B-PLS. The TPS warps (Fig. 8 and Fig. 9) show that most significantly integrated bones diverge in shape, rather than length, a pattern that mirrors the predominance of negative multiple-distance partial correlations. Relative changes of epiphyseal shape (reflected in asymmetric or irregular warp grid patterns between epiphyseal landmarks) and overall bone proportion (sturdiness vs. gracility; expressed through compressed or elongated warp grids along the bones) were involved in nearly all bone pairs with significant r-PLS.

\section{Rarefaction analyses (Fig. 10, Figs. S2, 3)}

Rarefaction analyses of the partial correlation and r-PLS coefficients in the two best-sampled species $-I$. obesulus $(n=56)$ and $P$. gunnii $(n=29)-$ revealed that very small sample sizes (under 20) have a high risk of inaccurate correlations/r-PLS values and unreliable EED or $p$ values, with a high likelihood of false negatives and in some cases false positives (Fig. 10 for a selection of results; Figs. S2-3). The rarefaction results also depended on whether the fullsample analyses were significant or not; in bone pairs that were significantly associated in the full sample, decreases in sample size resulted in increases of partial correlation values and their EED-values, and increasing variation around the mean. The r-PLS values remained 
relatively constant across sample sizes, but $p$-values increased substantially and varied far more in small sample sizes, with a very high chance of false negatives at sample sizes fewer than 20. In I. obesulus, partial correlations approximated the correlation and EED levels of the full sample around samples sizes of 20-30, whereas sample sizes around 30 already had substantial error around the mean in the r-PLS values. This suggests that our sampling of $P$. gunnii is only just sufficient, with a chance of false negatives in low-significance correlations., and a slightly higher expectation of result accuracy for the partial correlation analyses. correlation values and EED values tended to decrease or remain similar with increasing sample sizes, with very large variation at samples sizes of 10 ; however, these converged to the full-sample value at sample sizes of 20. In samples of 20 and below, the extremes of the error bars around the r-PLS $p$-values and EED scores frequently extended past the significance cut-offs, suggesting a risk of false positives in small sample sizes compared to large ones; however, our sample of $P$. gunnii appears sufficient to avoid this effect, with the partial correlation analysis again apparently more robust to small sample sizes.

\section{Principal components analysis}

PCAs of each bone Procrustes residuals datasets, and associated TPS warps, for each bone type are shown in Fig. 11. PC1 and 2 distinguished particularly between I. obesulus from $P$. gunnii, possibly due partly to the dominance of these two in the dataset. I. obesulus has more robust bones and wider epiphyses than $P$. gunnii, particularly in the humerus, metacarpals, and metatarsals. The other species mostly fall in between $I$. obesulus and $P$. 
gunnii, with congeneric species (i.e. P. gunnii and P. nasuta, I. macrourus and I. obesulus) not clustering particularly close on most PC plots.

\section{DISCUSSION}

\section{Bandicoot limb integration patterns do not follow expectations of developmental forelimb}

The length-only partial correlation patterns in our peramelid species (Fig. 4)

systematically differ from published results for other marsupials (Fig. 2), pointing to a systematic difference between the two. In particular, peramelemorphs, placentals, and monotremes have more significant correlations and more incidences of positive, length-only partial correlations between limbs and particularly between serial homologues than other marsupials. This is consistent with a weaker developmental constraint on peramelid forelimbs compared to other marsupials, as the climb to the pouch seems to reduce between-limb integration (Bennett and Goswami 2011; Kelly and Sears 2011). Furthermore, strong within-forelimb integration is one of the expected outcomes of the marsupial developmental constraint (Bennett and Goswami 2011; Kelly and Sears 2011), but there is only a single significant within-forelimb partial correlation in the entire peramelid sample. Indeed, peramelids $I$. obesulus and $P$. nasuta are unique among mammals to show no integration of length measurements within fore- or hind limbs at all. are positive (Fig. 4), while the multiple-distance dataset generates more numerous and nearly all negative significant partial correlations (Fig. 5). These appear to reflect opposing shape changes between bones, as observed in the thin-plate spline warp plots of the 2B-PLS analysis ("PLS warp plots" herein). For example, both humeral epiphyses of $I$. obesulus and 
P. gunnii widen laterally and compress proximo-distally as the radii become more gracile

(Fig. 8 and Fig. 9). This contradicts the prediction that the marsupial developmental constraint results in low within-forelimb divergence (Bennett and Goswami 2011; Kelly and Sears 2011, 2011) and in an absence of covariation between limbs (Bennett and Goswami 2011; Kelly and Sears 2011). With the caveats of low sample sizes in most published marsupial results (see below), our results therefore tentatively support the hypothesis that their low-intensity "slither" to the pouch leads to a reduction of biomechanical developmental constraints on the peramelid forelimb compared to other marsupials. This also adds to the evidence for a mostly biomechanical driver of the integration patterns seen in other marsupials, through selection on the developing forelimbs towards a climbingadapted shape (see also Sears 2004; Weisbecker et al. 2008; Linde-Medina and Diogo 2014).

\section{Bandicoot limb shape integration appears driven by function, not serial homology}

Peramelids display more between-limb and fewer within-limb length-only significant partial correlations than any other mammal in the comparative studies, suggesting more complex drivers of peramelid limb integration than a simple return to the serial homologydominated pattern (Young and Hallgrímsson 2005; Bennett and Goswami 2011; Kelly and Sears 2011). This is also supported by the abundance of divergent shape changes in significant multiple-distance partial correlations, biplots, and 2B-PLS associations. This is opposite to the expectation of integration arising from serial homology, which is thought to arise from related developmental programmes that specify similar skeletal growth patterns (Young and Hallgrímsson 2005). Serial homologues should thus covary not only in magnitude, but also direction (i.e. a pair of serially homologous bones should change in the same way), which is mostly not the case in peramelids. Function-related covariation explains the pattern of bandicoot limb integration much better, because selection for function can 
act on pairs of bones to a similar degree, with no restriction on how these bones co-vary in shape (Fischer et al. 2002; Schmidt and Fischer 2009; Diogo et al. 2013; Klingenberg 2014; Martín-Serra et al. 2015). We conclude that function must play a substantial role in the coevolution of limb bones relative to each other in bandicoots.

Bandicoots provide a useful assessment of potential functional selection pressures on integration patterns because they are ecologically similar with subtle differences. All bandicoots, including our best-sampled species ( $I$. obesulus and $P$. gunnii) use their hind limbs for half-bounding, and their forelimbs for scratch-digging (Strahan 2004; Weisbecker and Warton 2006; Warburton et al. 2013). However, the more fossorial I. obesulus turns over one-third more soil for its body weight (Fleming et al. 2013) and is the only peramelid thought to construct its own burrows (Long 2009). Our cross-species PCA plots reflect these different life histories, as the limb bones of $I$. obesulus are sturdier overall (a hallmark of fossoriality; Martín-Serra, Figueirido, and Palmqvist 2014)) and have wider epiphyses than $P$. gunnii, which is more gracile (generally associated with a hopping/cursorial habit; Lammers and German 2002; Weisbecker and Warton 2006; Schmidt and Fischer 2009). Changes in sturdiness relative to other bones also dominate the 2B-PLS results in $I$. obesulus, particularly for the humerus, metacarpal, and metatarsal, which are all employed in digging (Polly 2007; Warburton et al. 2013). In addition, several significant 2B-PLS correlations in $P$. gunnii - particularly those involving the humerus, femur, and radius - involve a bending of the epiphyseal area (Fig. 9), which is frequently seen in hopping mammals and may allow a more efficient pre-jump crouching posture (McGowan et al. 2008).

It is also possible that the significant allometric signal in our dataset is reflected in the shape changes, with differently-sized animals presumably experiencing slightly different 
biomechanical loads on their skeleton. Notably, partial correlation and 2B-PLS results for $I$. obesulus and $P$. gunnii have similar signs and significance levels, but their underlying physical manifestations as seen in the 2B-PLS plots are markedly different. For example, the humerus of $I$. obesulus widens at the head and compresses at the base with increasing bending and head torsion of the tibia; by contrast, the humerus in $P$. gunnii compresses at the head and widens at the base with increasing slenderness of the tibia base. The evolution of limb adaptation might be very fast, as is suggested by several significant differences in the bone shapes of individuals from different locations. Fast local adaptation to soil conditions is common in populations of fossorial mammals (Marcy et al. 2016), so that this result is not unexpected. However, the Procrustes shape differences between left and right radii in $I$. obesulus and $P$. gunnii might even hint at individually acquired shapes related to the "handedness" of the animal, an intriguing possibility warranting further research. - is the only species with three positive between-limb multiple length partial correlations. Counter to previous assumptions (Weisbecker et al. 2008; Cooper and Steppan 2010), video footage (Tait 2001) shows that bilby neonates engage in an extensive climb to the pouch. A functional explanation for this difference is thus more likely, as bilbies are more cursorial and fossorial than peramelids (Warburton et al. 2013); more data are required to understand this exception, given the low sample size of bilbies in this study $(n=10)$. 
In addition to indicating an overall strong signal of functional selection on peramelid limbs, the divergent partial correlation signs in the length-only versus multiple-distance comparisons also suggest differences between diaphyseal (which dominate the length-only data) and epiphyseal (which dominate the multiple-distance data) covariation. This is substantiated by a considerable contribution of epiphyseal shape in the warp plots of most significant 2B-PLS comparisons. A follow-up partial correlation analysis using just epiphyseal data (the multiple-distance index minus the diaphyseal length measurement) bears little resemblance to length-only or multiple-distance analyses (Fig. S4, Table S10), suggesting that epiphyseal shape indeed holds different information from data that incorporate length. These differences may relate to a developmental mechanism: epiphyses of mammalian limb long bones ossify much later than - almost independently from - the diaphyses, and in marsupials they ossify exclusively postnatally (Hamrick 1999). Mammalian diaphyses and epiphyses join very late or not at all, and do so independently of locomotor habit (Geiger et al. 2014). In contrast, epiphyseal joint surface development is extensively influenced by mechanical stresses applied on the joints (Carter et al. 1998; Hamrick 1999; Green et al. 2012; Sylvester 2015). This matches the fine-grained resolution on locomotor behaviour reflected by mammalian epiphyseal shape (Walmsley et al. 2012; Fabre et al. 2015) and integration patterns (Fabre et al. 2014). It is thus possible that epiphyseal shape is extensively driven by function, while the developmentally decoupled, early-ossifiying diaphyses are more likely to reveal impacts on earlier development, such as the marsupial climb to the pouch. This effect may be even more pronounced in many marsupials, including peramelemorphs, whose epiphyses of the fore- and hind limb bones never close (other 
parts of the skeleton - e.g. the scapula or pelvis - ossify to varying degrees in

peramelemorphs and other marsupials) (Geiger et al. 2014). The traditional use of length measurements, which reflect diaphyseal shape more than epiphyseal shape, might thus provide a more relevant assessment of the impact of pre-or perinatal events such as the marsupial climb to the pouch.

\section{Small sample sizes produce false negatives and positives}

Our rarefaction analyses show that very small sample sizes (20 or less) may be more detrimental to the reliability of integration studies than previously thought, as they frequently produce false negatives and have a substantial risk of false positives, particularly in the 2B-PLS analyses. This is despite relatively high repeatability values derived from random skewers analysis (Marroig and Cheverud 2001; Young and Hallgrímsson 2005) in our study and others (Table S3; Bennett and Goswami 2011; Kelly and Sears 2011). These results are under Cheverud's (1988) recommendation of a sample size of around 40 for reliable assessments of integration (Polly 2005), but still suggest that distance- and Procrustes-shape integration analyses with low sample sizes need to be interpreted with caution (Cheverud 1988; Goswami and Polly 2010; Goswami et al. 2014). For example, only two of our species and three other marsupials (Monodelphis domestica, Didelphis virginiana, and Dromiciops gliroides) studied to date (Kelly and Sears 2011) have sample sizes of 30 and more and thus an expectation of providing accurate results. 
Our addition of bandicoots to the existing body of literature on mammalian limb integration lends some support to the hypothesis that the developmental constraint on the marsupial forelimb acts at least partially through a relatively immediate biomechanical or resource allocation impact at birth. However, we also find that small-sample sizes - such as those presented in most previous studies - might be unsuitable in providing reliable assessments of limb bone integration. Better-sampled datasets across marsupials, incorporating length and proportion data, are required to further assess the relative impact of genetic developmental constraint versus patterns of functional integration on marsupial bone shape and proportions. Further investigations of the bilby - the sister group of peramelids, which displays an extensive climb to the pouch at birth - are particularly interesting, since even our small sample of this species suggests intriguing patterns that diverge from all other mammals sampled so far. In addition, differentiating between diaphyseal and epiphyseal shape covariation might be useful in future studies to disentangle the functional versus developmental causes of shape integration (Diogo et al. 2013; Geiger et al. 2014). Given the comparatively complicated computations required for analyses of integration, it also appears important to continue investing in more easily assessable observations of the actual animals, e.g. in terms of locomotor behaviour, gross morphology/myology (Diogo and Molnar 2014), and development. Lastly, the matching of existing knowledge on skeletal functional morphology with the visual output of 2B-PLS integration analysis represents a promising avenue of providing a more detailed evolutionary narrative of mammalian skeletal evolution, from the population-level to macroevolutionary comparisons. 


\section{ACKNOWLEDGEMENTS}

We thank the museum curators and collection managers S. Ingleby (Australian Museum), T. Gordon (Launceston Museum), H. Janetzki (Queensland Museum), J. Waldock (Western Australia Museum) D. Stemmer (South Australia Museum) and K. Rowe (Victoria Museum) for granting access to their collections. We also would like to acknowledge the members of the Weisbecker Laboratory for their assistance throughout this study. We thank Simon Blomberg for providing statistical advice and the covariance repeatability code. We are grateful to Artemis International for providing footage of a bilby birth. V. Weisbecker was supported by Discovery Early Career Award (DE120102034,) A. Marcy by the AustralianAmerican Fulbright Commission (2014 U.S. Postgraduate Fulbright Scholarship).

\section{REFERENCES}

Adams, D. C. and Otárola-Castillo, E. 2013. geomorph: an r package for the collection and analysis of geometric morphometric shape data. Methods Ecol. Evol. 4: 393-399.

Bennett, C., V. and Goswami, A. 2011. Does developmental strategy drive limb integration in marsupials and monotremes? Mamm. Biol. 76: 79-83.

Bininda-Emonds, O. R., Jeffery, J. E., Sánchez-Villagra, M. R., Hanken, J., Colbert, M., Pieau, C., Selwood, L., Ten Cate, C., Raynaud, A., Osabutey, C. K., and Richardson, M. K. 2007. Forelimb-hindlimb developmental timing changes across tetrapod phylogeny. BMC Evol. Biol. 7: 182.

Carter, D. R., Mikić, B., and Padian, K. 1998. Epigenetic mechanical factors in the evolution of long bone epiphyses. Zool. J. Linn. Soc. 123: 163-178.

Cheverud, J. M. 1982. Phenotypic, genetic, and environmental morphological integration in the cranium. Evolution 36: 499-516. 
Cheverud, J. M. 1988. A Comparison of Genetic and Phenotypic Correlations. Evolution 42: 958-968.

Cheverud, J. M. and Marroig, G. 2007. Comparing covariance matrices: random skewers method compared to the common principal components model. Genet. Mol. Biol. 30: 461-469.

Chew, K. Y., Shaw, G., Yu, H., Pask, A. J., and Renfree, M. B. 2014. Heterochrony in the regulation of the developing marsupial limb. Dev. Dynam. 243: 324-338.

Cooper, W. J. and Steppan, S. J. 2010. Developmental constraint on the evolution of marsupial forelimb morphology. Aust. J. Zool. 58: 1-15.

Cuvier, G.1800. Leçons d'Anatomie Comparée. Paris: Baudouin, Paris.

Diogo, R., Linde-Medina, M., Abdala, V., and Ashley-Ross, M. A. 2013. New, puzzling insights from comparative myological studies on the old and unsolved forelimb/hindlimb enigma. Biological Reviews 88: 196-214.

Diogo, R. and Molnar, J. 2014. Comparative anatomy, evolution, and homologies of tetrapod hindlimb muscles, comparison with forelimb muscles, and deconstruction of the forelimb-hindlimb serial homology hypothesis. Anat. Rec. 297: 1047-1075.

Diogo, R. and Ziermann, J. 2015. The End of an Old Dogma with Crucial Implications for Medical and Biology Students and for Medicine: regenerative, Developmental, Paleontological and Evolutionary Studies Contradict the Fore-hindlimb Serial Homology. FASEB J. 29.

Fabre, A. C., Cornette, R., Goswami, A., and Peigné, S. 2015. Do constraints associated with the locomotor habitat drive the evolution of forelimb shape? a case study in musteloid carnivorans. J. Anat. 226: 596-610. 
Fabre, A. C., Goswami, A., Peigné, S., and Cornette, R. 2014. Morphological integration in the forelimb of musteloid carnivorans. J. Anat. 225: 19-30.

Fischer, M. S., Schilling, N., Schmidt, M., Haarhaus, D., and Witte, H. 2002. Basic limb kinematics of small therian mammals. J. Exp. Biol. 205: 1315-1338.

Flores, D. A., Abdala, F., and Giannini, N. P. 2013. Post-weaning cranial ontogeny in two bandicoots (Mammalia, Peramelomorphia, Peramelidae) and comparison with carnivorous marsupials. Zoology 116: 372.

Geiger, M., Forasiepi, A. M., Koyabu, D., and Sánchez-Villagra, M. R. 2014. Heterochrony and post-natal growth in mammals - an examination of growth plates in limbs. J. Evol. Biol. 27: 98-115.

Gemmell, R. T., Veitch, C., and Nelson, J. 2002. Birth in marsupials. Comp. Biochem. Physiol. B 131: 621-630.

Goodall, C. 1991. Procrustes methods in the statistical analysis of shape. Journal of the Royal Statistical Society. Series B (Methodological): 285-339.

Goswami, A. and Polly, P. D. 2010. Methods for studying morphological integration and modularity Paleo. Soc. 16: 213-243.

Goswami, A., Smaers, J. B., Soligo, C., and Polly, P. D. 2014. The macroevolutionary consequences of phenotypic integration: from development to deep time. Phil. Trans. R. Soc. Lon. B Biol. Sci. 369: 20130254-20130254.

Goswami, A., Weisbecker, V., and Sánchez-Villagra, M. R. 2009. Developmental modularity and the marsupial-placental dichotomy. J. Exp. Zool. B Mol. Dev. Evol. 312B: 186-95.

Green, D. J., Richmond, B. G., and Miran, S. L. 2012. Mouse shoulder morphology responds to locomotor activity and the kinematic differences of climbing and running. J. Exp. Zool. B Mol. Dev. Evol. 318: 621-638. 
Greenacre, M. J.2010. Biplots in Practice. Fundacion BBVA / BBVA Foundation, Spain.

Hammer, $\varnothing$. and Harper, D. A. T.2006. Paleontological data analysis. Blackwell Pub, Malden, MA.

Hamrick, M. W. 1999. Development of epiphyseal structure and function in Didelphis virginiana (Marsupiala, Didelphidae). J. Morph. 239: 283-296.

Hughes, R. L. and Hall, L. S. 1993. Early development and embryology of the platypus. Philos. Trans. R. Soc. Lond., B, Biol. Sci. 353: 1101-1114.

Jungers, W. L., Falsetti, A. B., and Wall, C. E. 1995. Shape, relative size, and size-adjustments in morphometrics. Am. J. Phys. Anthropol. 98: S137-S161.

Kelly, E. M. and Sears, K. E. 2011. Limb specialization in living marsupial and eutherian mammals: constraints on mammalian limb evolution. J. Mammal. 92: 1038-1049.

- - . 2011. Reduced phenotypic covariation in marsupial limbs and the implications for mammalian evolution. Biol. J. Linn. Soc. 102: 22-36.

Keyte, A. and Smith, K. K. 2012. Heterochrony in somitogenesis rate in a model marsupial, Monodelphis domestica. Evol. Dev. 14: 93-103.

Keyte, A. L. and Smith, K. K. 2010. Developmental origins of precocial forelimbs in marsupial neonates. Development 137: 4283-4294.

Kingsmill, E. 1962. An investigation of criteria for estimating age in the Marsupials Trichosurus vulpecula Kerr and Perameles nasuta Geoffroy. Aust. J. Zool. 10: 597-616.

Kirsch, J. A. 1977. Biological aspects of the marsupial-placental dichotomy: a reply to Lillegraven. Evolution 31: 898-900.

Klingenberg, C. P. 2014. Studying morphological integration and modularity at multiple levels: concepts and analysis. Phil. Trans. R. Soc. Lon. B Biol. Sci. 369: 2013024920130249. 
Lammers, A. R. and German, R. Z. 2002. Ontogenetic allometry in the locomotor skeleton of specialized half-bounding mammals. J. Zool. 258: 485-495.

Lillegraven, J. A. 1975. Biological considerations of the marsupial-placental dichotomy. Evolution 29: 707-722.

Linde-Medina, M. and Diogo, R. 2014. Do correlation patterns reflect the role of development in morphological evolution? Evol. Biol. 41: 494-502.

Magwene, P. M. 2001. New tools for studying integration and modularity. Evolution 55: $1734-1745$.

Marcy, A. E., Hadly, E. A., Sherratt, E., Garland, K., and Weisbecker, V. 2016. Getting a head in hard soils: Convergent skull evolution and divergent allometric patterns explain shape variation in a highly diverse genus of pocket gophers (Thomomys). BMC Evolutionary Biology 16: 207.

Marroig, G. and Cheverud, J. M. 2001. A comparison of phenotypic variation and covariation patterns and the role of phylogeny, ecology, and ontogeny during cranial evolution of new world monkeys. Evolution 55: 2576-2600.

Martín-Serra, A., Figueirido, B., and Palmqvist, P. 2014. A three-dimensional analysis of the morphological evolution and locomotor behaviour of the carnivoran hind limb. BMC Evol. Biol. 14: 129-129.

Martín-Serra, A., Figueirido, B., Pérez-Claros, J. A., and Palmqvist, P. 2015. Patterns of morphological integration in the appendicular skeleton of mammalian carnivores. Evolution 69: 321-340.

McGowan, C. P., Skinner, J., and Biewener, A. A. 2008. Hind limb scaling of kangaroos and wallabies (superfamily Macropodoidea): implications for hopping performance, safety factor and elastic savings. J. Anat. 212: 153-163. 
Mitchell, K. J., Pratt, R. C., Watson, L. N., Gibb, G. C., Llamas, B., Kasper, M., Edson, J., Hopwood, B., Male, D., and Armstrong, K. N. 2014. Molecular phylogeny, biogeography, and habitat preference evolution of marsupials. Mol. Biol. Evol.: msu176.

Oksanen, J., Kindt, R., Legendre, P., O'Hara, B., Stevens, M. H. H., Oksanen, M. J., and Suggests, M. 2007. The vegan package. Community ecology package: 631-637.

Owen, R.1849. On the nature of limbs : a discourse delivered on Friday, February 9, at an evening meeting of the Royal Institution of Great Britain.

Polly, P. D. 2005. Development and phenotypic correlations: the evolution of tooth shape in Sorex araneus. Evolution \& Development 7: 29-41.

Polly, P. D. 2007 Limbs in mammalian evolution. In Fins into Limbs: Evolution, Development and Transformation, edited by B. Hall. Chicago: The University of Chicago Press Books.

R Development Core Team. $R$ : A language and environment for statistical computing. $v$. 3.2.3. R Foundation for Statistical Computing 2016. Available from http://www.Rproject.org.

Reese, S., Pfuderer, U. R., Bragulla, H., Loeffler, K., and Budras, K. D. 2001. Topography, Structure and Function of the Patella and the Patelloid in Marsupials. Anat. Histol. Embryol. 30: 289-294.

Rohlf, F. J. and Corti, M. 2000. Use of two-block partial least-squares to study covariation in shape. Syst. Biol. 49: 740-753.

Rohlf, J. 2013. TPSdig, v. 2.17: NY State University at Stony Brook

Rohlf, J. and Slice, D. 1990. Extensions of the procrustes method for the optimal superimposition of landmarks. Syst. Biol. 39: 40-59. 
Sánchez-Villagra, M. R. 2002. Comparative patterns of postcranial ontogeny in therian mammals: an analysis of relative timing of ossification events. J. Exp. Zool. 294: 264273.

Sánchez-Villagra, M. R., Goswami, A., Weisbecker, V., Mock, O. B., and Kuratani, S. 2008. Conserved relative timing of cranial ossificaiton patterns in early mammalian evolution. Evol. Devo. 10: 519-530.

Schaefer, J., Opgen-Rhein, R., and Strimmer, K. 2013. corpcor: efficient estimation of covariance and (partial) correlation. $R$ package version 1.

Schmidt, M. and Fischer, M. S. 2009. Morphological integration in mammalian limb proportions: dissociation between function and development. Evolution 63: 749-766.

Sears, K. E. 2004. Constraints on the morphological evolution of marsupial shoulder girdles. Evolution 58: 3253-2370.

- - . 2009. Differences in the Timing of Prechondrongenic Limb Development in Mammals: The Marsupial- Placental Dichotomy Resolved. Evolution 63: 2193-2200.

Sears, K. E., Capellini, T. D., and Diogo, R. 2015. On the serial homology of the pectoral and pelvic girdles of tetrapods. Evolution 69: 2543-2555.

Sears, K. E., Patel, A., Hubler, M., Cao, X., Vandeberg, J. L., and Zhong, S. 2012. Disparate Igf1 expression and growth in the fore- and hind limbs of a marsupial mammal (Monodelphis domestica). J. Exp. Zoo. B Mol. Dev. Evol. 318: 279-93.

Strahan, R.2004. The mammals of Australia. Vol. 2nd reprint. Reed New Holland, Sydney.

Sylvester, A. D. 2015. Femoral Condyle Curvature is Correlated with Knee Walking Kinematics in Ungulates. Annat. Rec. 298: 2039-2050.

Szalay, F. S.1994. Evolutionary history of the marsupials and an analysis of osteological characters. Cambridge University Press, Cambridge. 
Tait, C. 2001. Return to Eden. Perth Australian Film Fianance Corporation Ltd.

Wagner, G. P. and Altenberg, L. 1996. Complex adaptations and the evolution of evolvability. Evolution 50: 967.

Walmsley, A., Elton, S., Louys, J., Bishop, L. C., and Meloro, C. 2012. Humeral epiphyseal shape in the felidae: The influence of phylogeny, allometry, and locomotion. J. Morph. 273: 1424-1438.

Warburton, N. M., Gregoire, L., Jacques, S., and Flandrin, C. 2013. Adaptations for digging in the forelimb muscle anatomy of the southern brown bandicoot (Isoodon obesulus) and bilby (Macrotis lagotis). Aust. J. Zool. 61: 402-419.

Weisbecker, V. 2011. Monotreme ossification sequences and the riddle of mammalian skeletal development. Evolution 65: 1323-1335.

Weisbecker, V. 2015 Are Monotremes primitive and Marsupials inferior? In Marsupials and Monotremes - Enigmatic Mammals., edited by A. Klieve and L. Hogan. New York: Nova Science Publishers.

Weisbecker, V., Goswami, A., Wroe, S., and Sánchez-Villagra, M. R. 2008. Ossification heterochrony in the therian postcranial skeleton and the marsupial-placental dichotomy. Evolution 62: 2027-41.

Weisbecker, V. and Warton, D. I. 2006. Evidence at hand: Diversity, functional implications, and locomotor prediction in intrinsic hand proportions of diprotodontian marsupials. J. Morph. 267: 1469-1485.

Williams, W. D., Walton, D. W., Australia. Bureau of, F., and Fauna.1987. Fauna of Australia. A.G.P.S, Canberra.

Young, N. and Hallgrímsson, B. 2005. Serial Homology and the Evolution of Mammalian Limb Covariation Structure. Evolution 59: 2961-2704. 
664 


\section{FIGURE LEGENDS}

Fig. 1. Hypothesized patterns of within-limb (black), between-limb (grey) and functional (dashed) integration in mammals. $\mathrm{H}=$ humerus; $\mathrm{R}=$ radius; $\mathrm{MC}=$ metacarpal $\mathrm{IV} ; \mathrm{F}=$ femur; $\mathrm{T}=$ tibia; $\mathrm{MT}=$ metatarsal IV. Lines represent those limb bones expected to share the greatest degree of limb bone integration.

Fig. 2. Line graphs representing the limb bones that were significantly correlated in published length-only partial correlation matrices of placental, marsupial and monotreme species. Bone abbreviations as in Fig. 1; $\mathrm{n}$ is sample size. From two studies on monotremes, displayed are the results with the largest sample sizes (Kelly and Sears, 2011/echidna; Bennett and Goswami, 2011, /platypus).

Fig. 3. Data acquisition and analysis workflows for this study. Grey cells indicate preliminary assessments of allometry and population sub-structure, rarefaction, and random skewers analysis.

Fig. 4. Line graphs of significantly correlated the limb bones for length-only partial correlation analyses. Values adjacent to lines are the respective correlation values between the bones. Abbreviations as in Fig. 1. Black lines represent within-limb bone correlations and grey limb represent between-limb bone correlations.

Fig. 5. Line graphs of significantly correlated the limb bones for multiple-distance partial correlation analyses. Dashed line is a representative of near significance. Abbreviations as in Fig. 1.

Fig. 6. Multiple-distance Principal Component Biplot analysis for A) I. obesulus, B) P. gunnii, C) P. nasuta, D) I. macrourus and E) M. lagotis limb bones. Arrows indicate the bone vectors. PC 1= principal component 1 and $\mathrm{PC} 2=$ Principal component 2 , and associated percentages represent the eigenvalues for each principal component. Three asterisks $(* * *)$ denote $p$-values $\leq 0.0001$, two 
$\left({ }^{* *}\right)$ denotes P-values $\leq 0.001,1\left(^{*}\right)$ denotes P-values $\leq 0.01$, period $($.$) denotes \mathrm{P}$-values $\leq 0.05$. See also Table S6.

Fig. 7. Line graphs of significantly correlated limb bones in the 2B-PLS analyses.. Values adjacent to lines are the respective correlation coefficients between the bones. Bone abbreviations as in Fig.

1.

Fig. 8. TPS warps depicting the morphological associations between each pair of bones compared by 2B-PLS analyses of Isoodon obesulus. The grid shape is the maximum amount of shape deformation from the mean shape along the first PLS axes. Abbreviations as in Fig. 1.

Fig. 9. TPS warps depicting the morphological associations between each pair of bones compared by 2B-PLS analyses of Perameles gunnii. The grid shape is the maximum amount of shape deformation from the mean shape along the first PLS axes. Abbreviations as in Fig. 1.

Fig. 10. Rarefaction analysis: the effect of small sample sizes, as tested by rarefaction analysis, on results of partial correlation (top) and 2B-PLS (bottom). Note the much larger variation in EED and $p$-values in smaller samples, and the tendency of both EED and $p$-values to decrease with increasing sample size.

Fig. 11. PCA plot of peramelemorph species limb bone shape variation for the A) humerus, B) femur, C) radius, D) tibia, E) metacarpal and F) metatarsal bones. Orange, l.obesulus, pink, $P$. gunnii, green, I. macrourus, blue, M. lagotis and yellow, P. nasuta. TPS warps represent bone shape described by the minima and maxima of PC1, with the grid representing the mean shape. The percentage of variation described by each PC axis is given in axis labels. 
FIGURES 
A. Ancestral Mammalian Condition

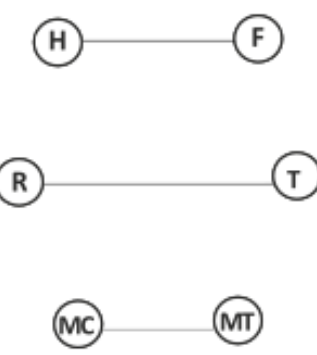

B. Developmental Constraint (Marsupials)

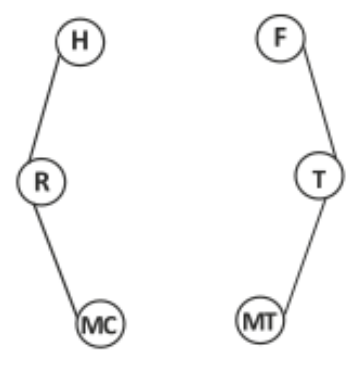

C. Reversal (Bandicoots)

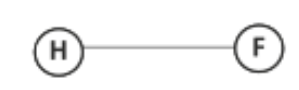

(R) $(\mathrm{T}$

(MC) (MT)
D. Functional Integration (Tetrapods)

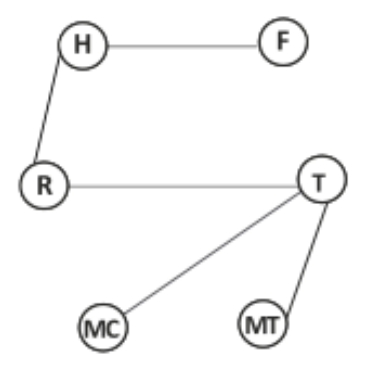

Fig 1. 


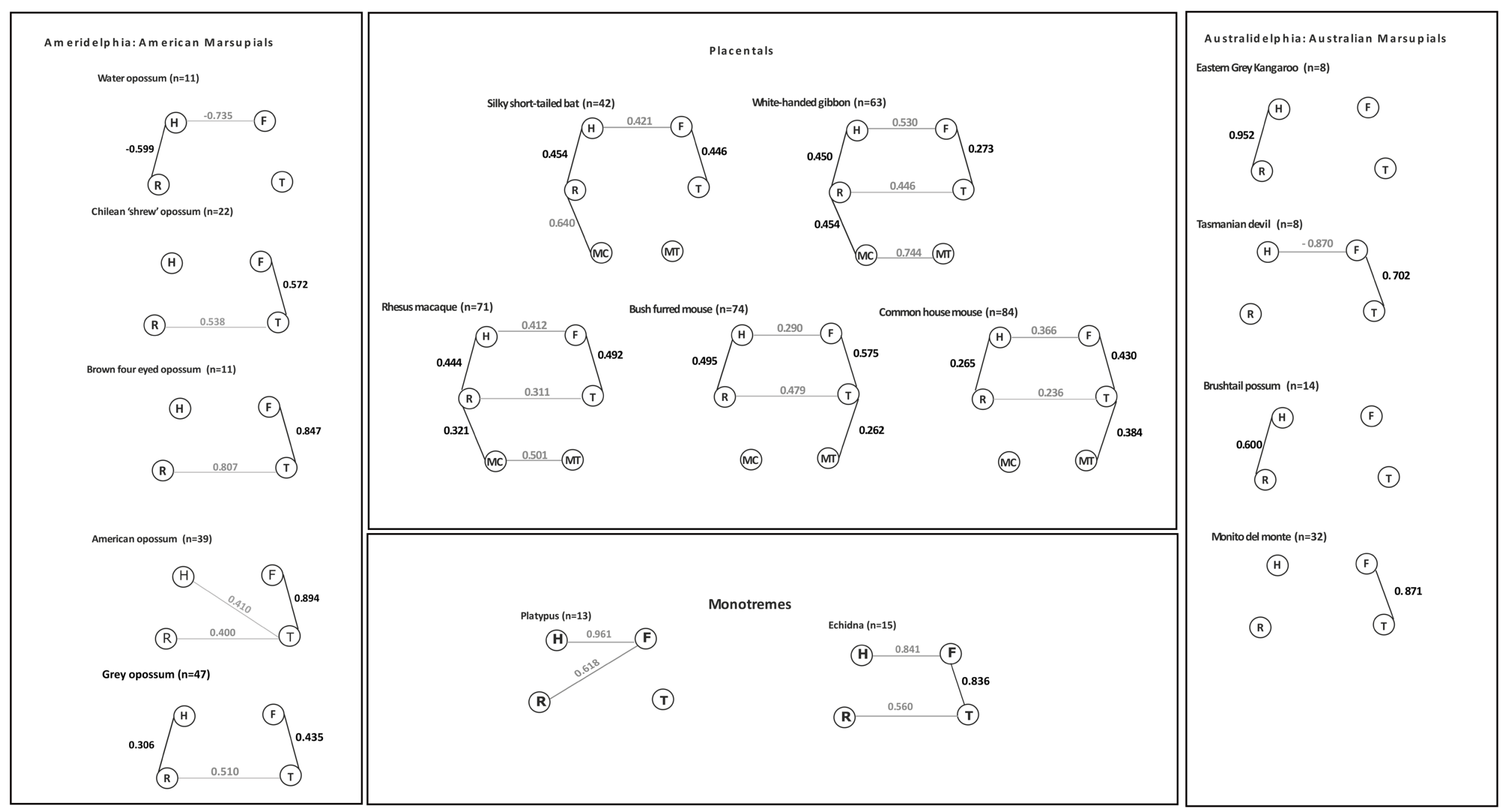

Fig 2. 

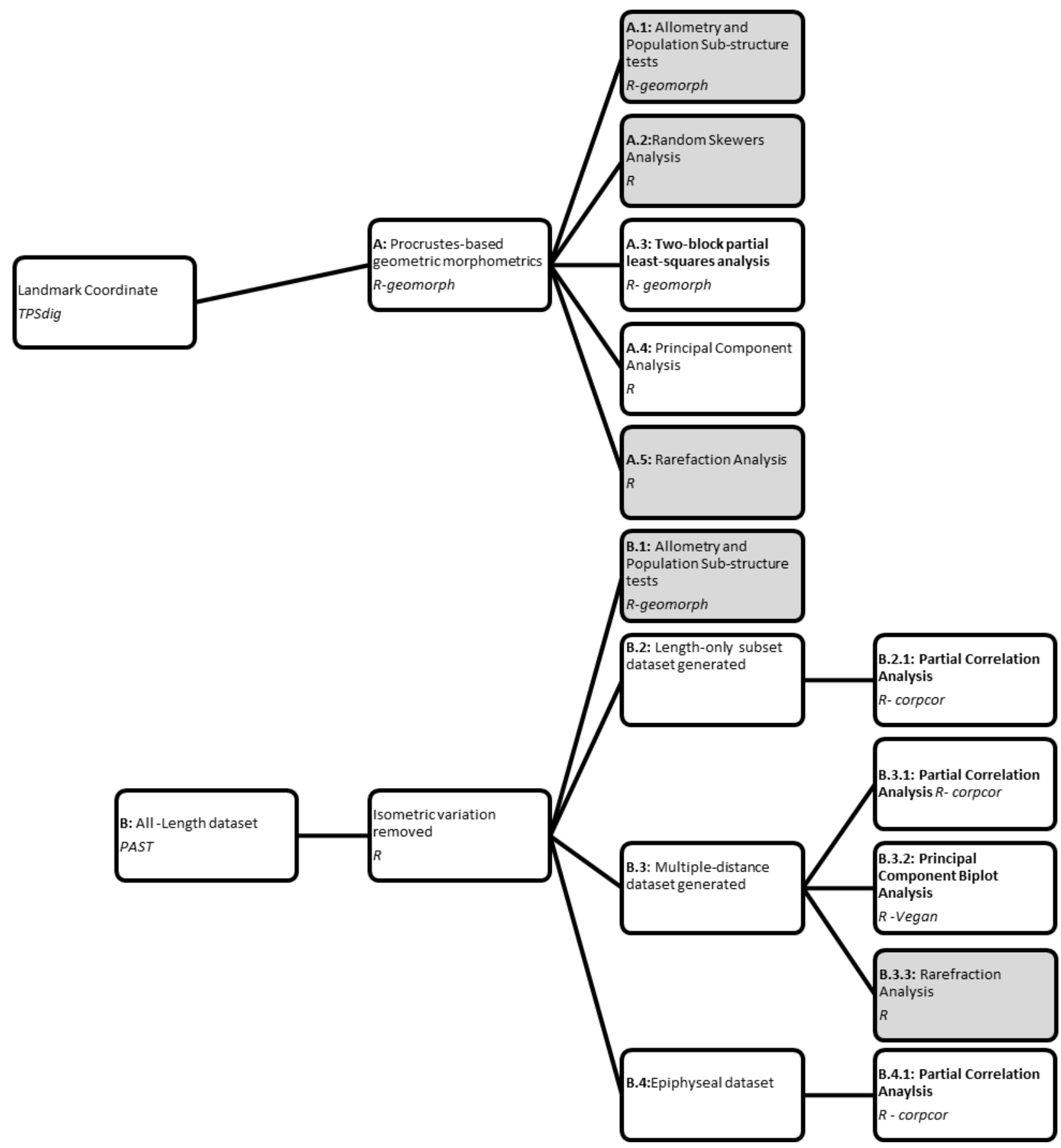

Fig. 3 


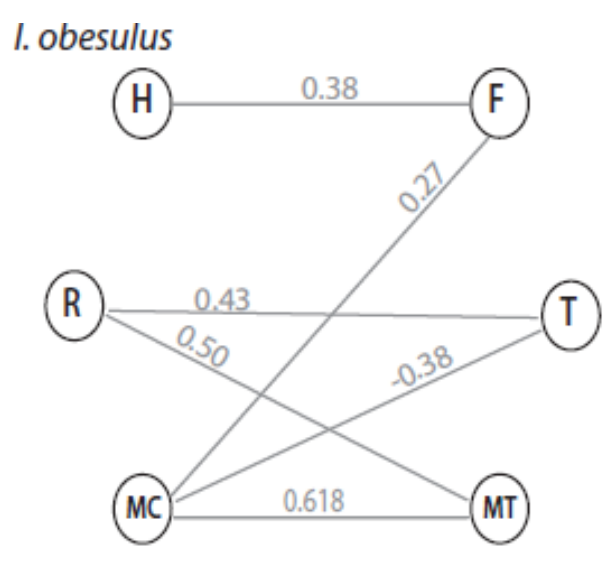

r.gunnII
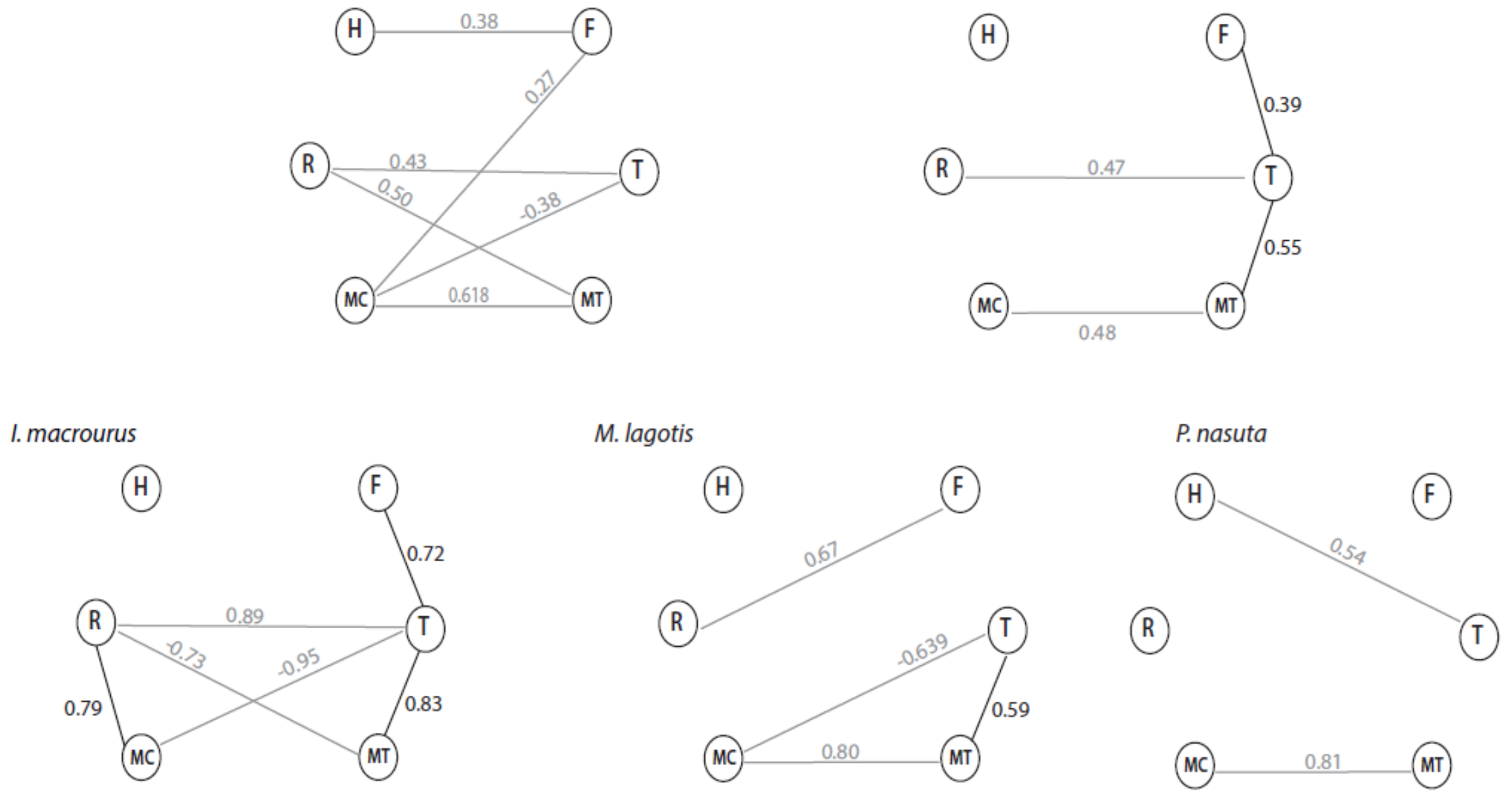

Fig. 4. 

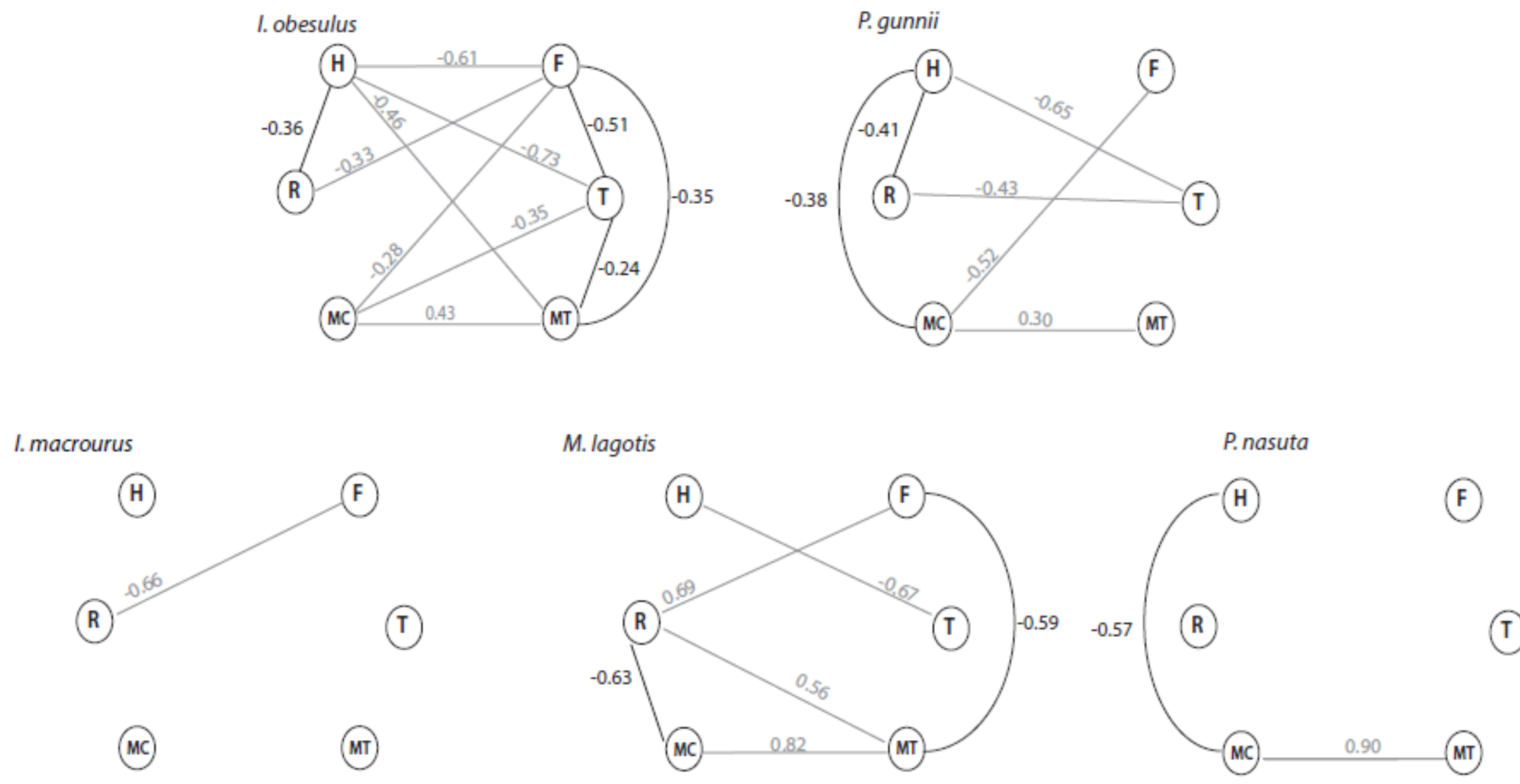

Fig. 5. 

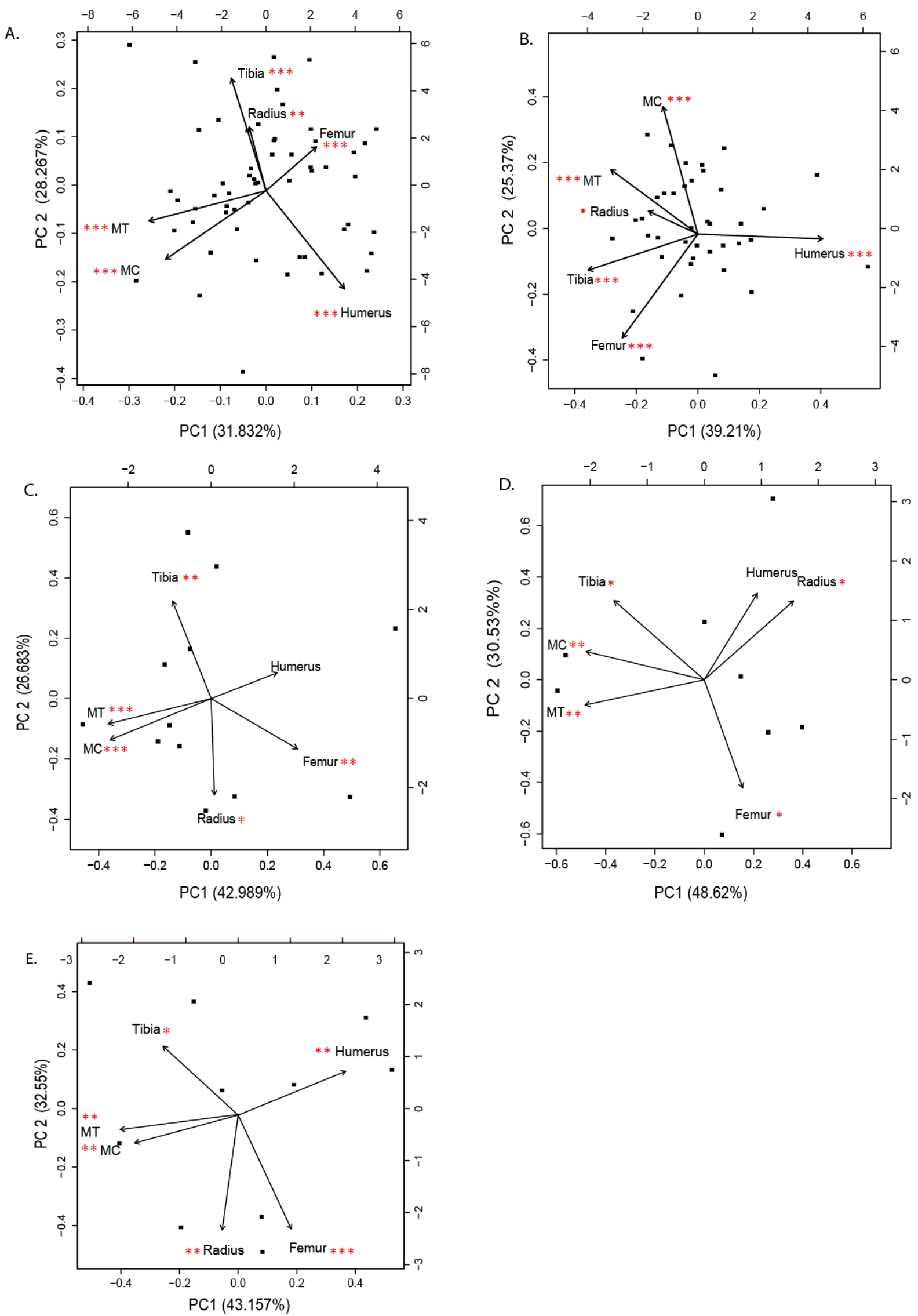

Fig. 6. 

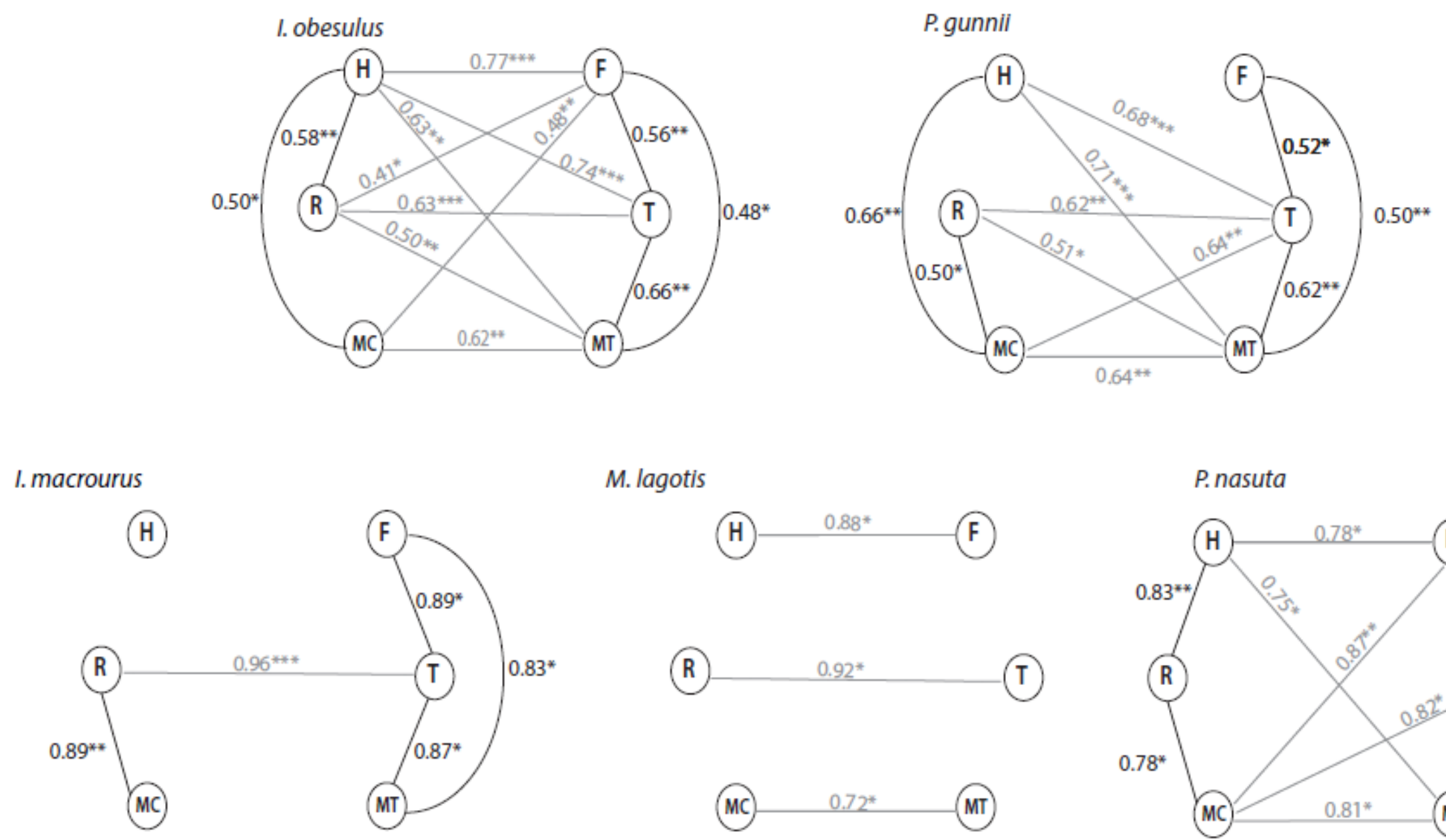

M. lagotis
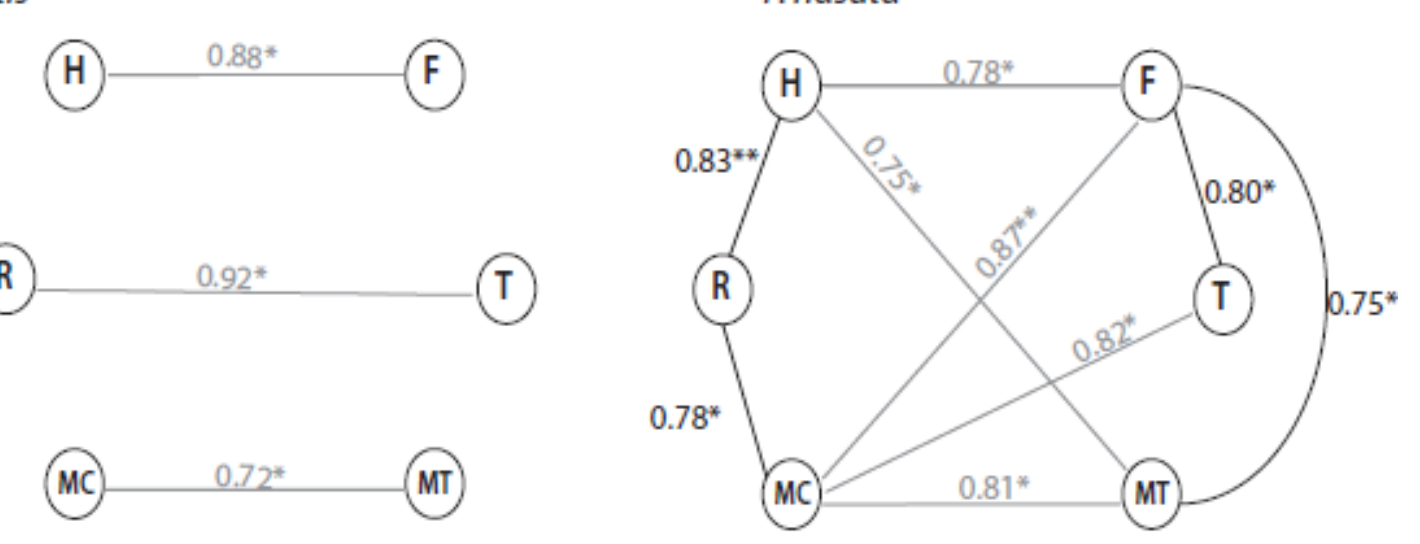

Fig. 7. 


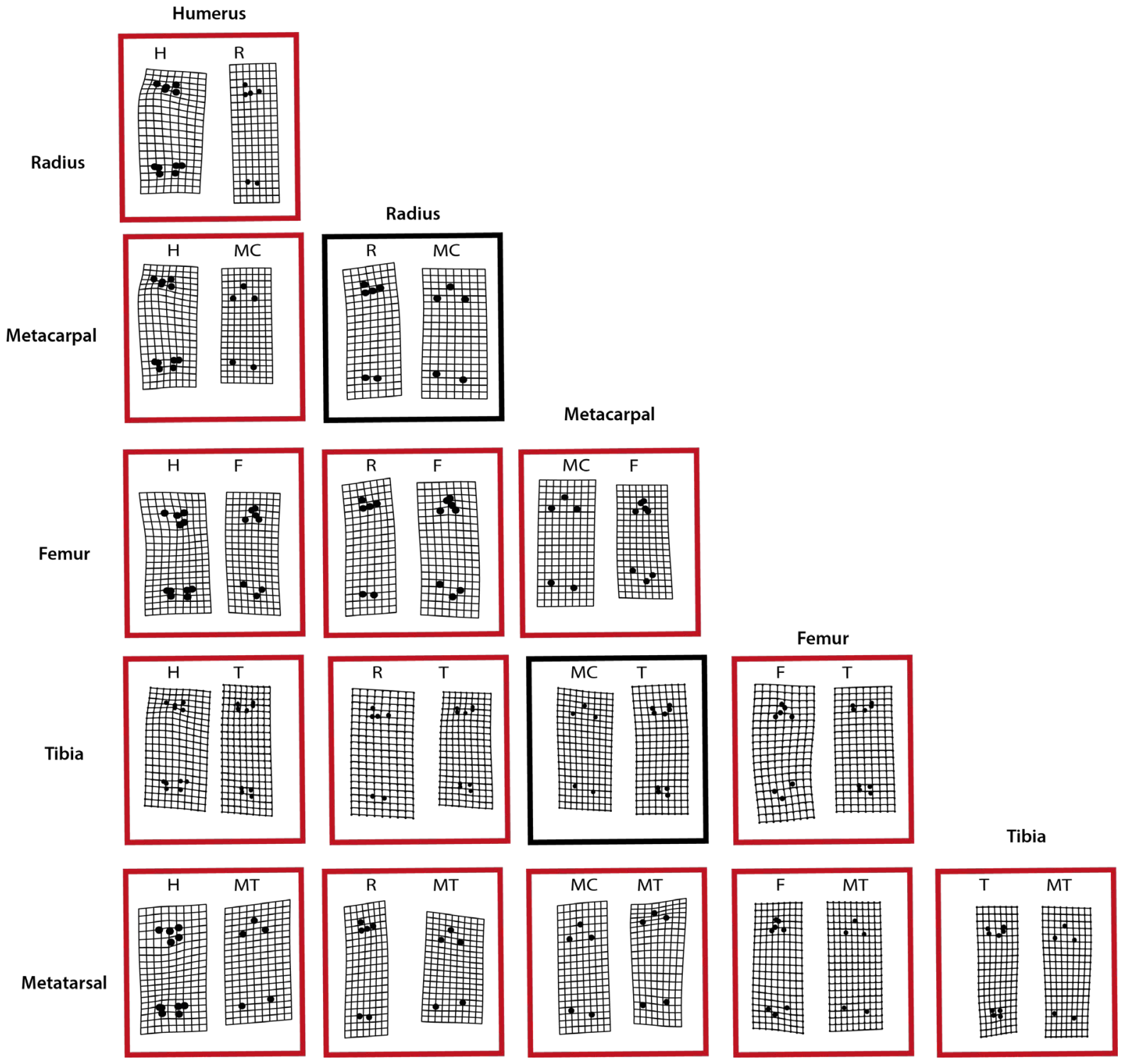

Fig. 8. 


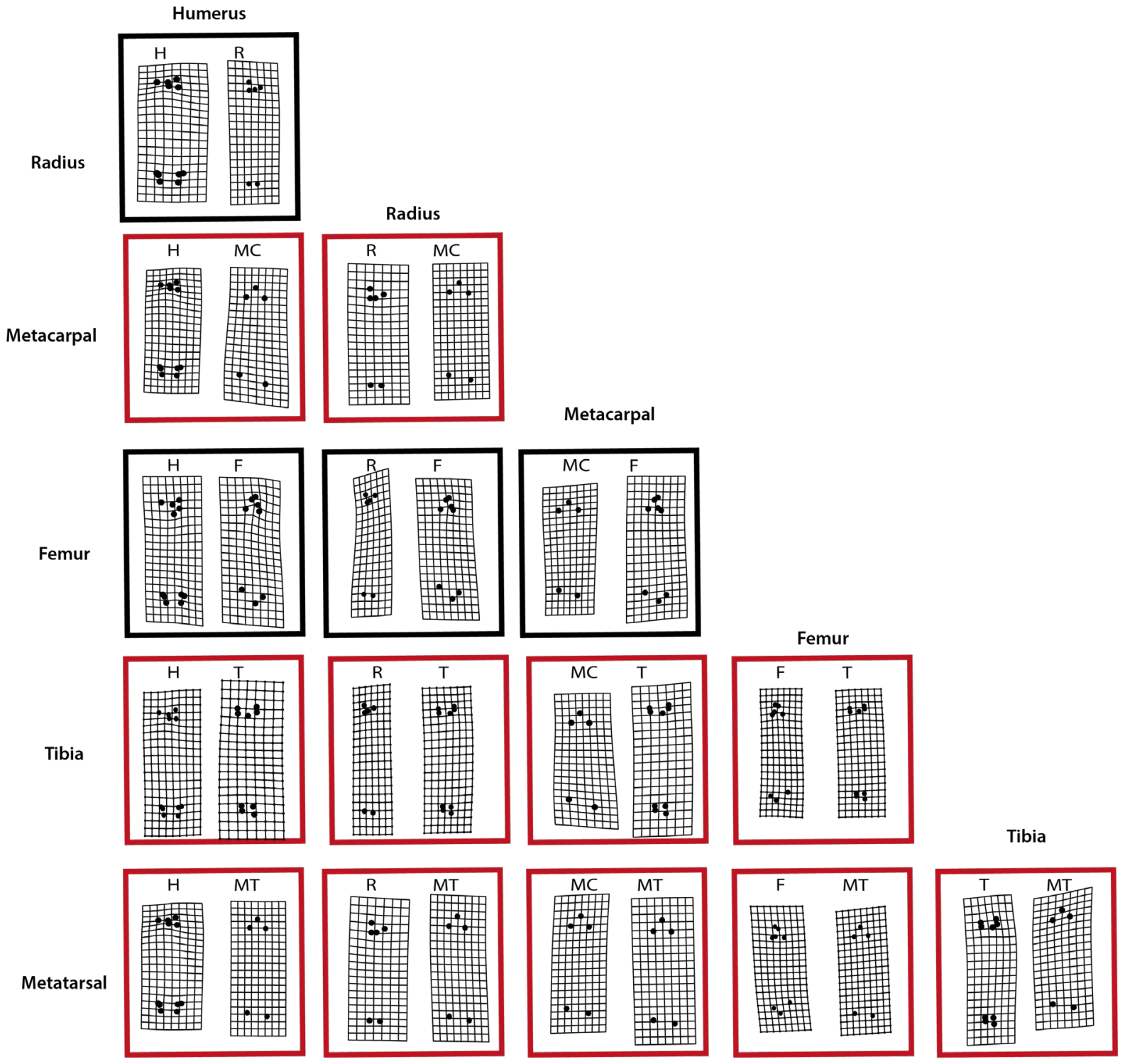

Fig. 9. 
Fig. 10.
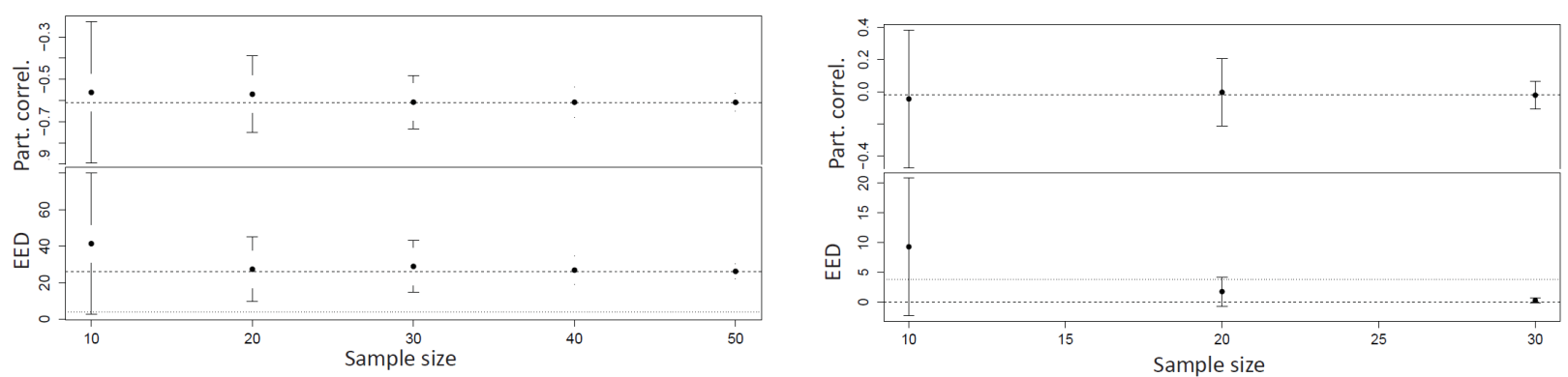

I.obesulus, Humerus-Femur: partial correl.=-0.61, EED=26.1
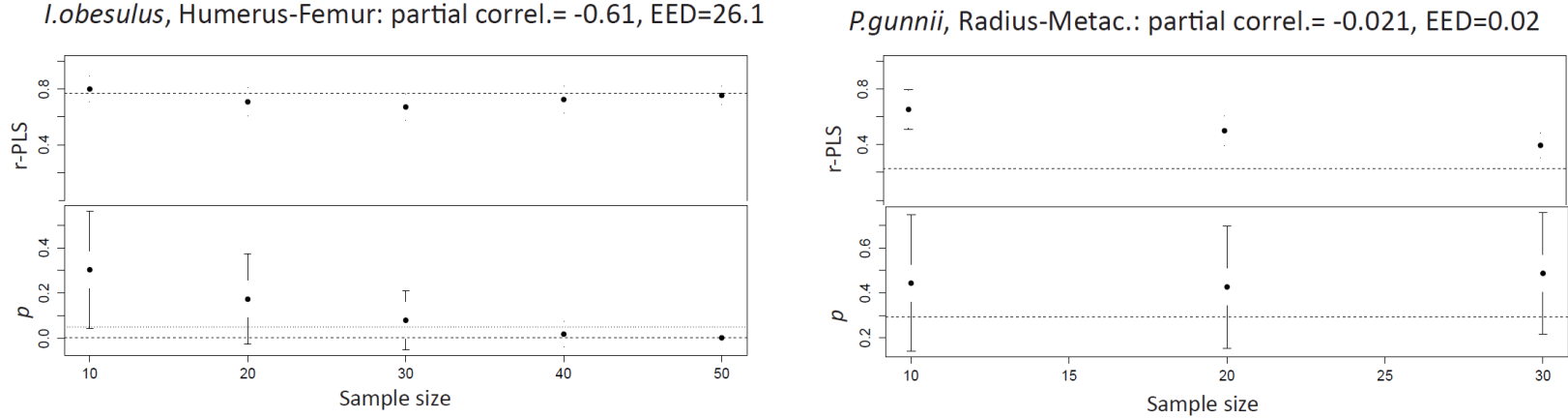

P. gunnii, Radius-Femur: r-PLS: $0.29 ; p=0.226$ 


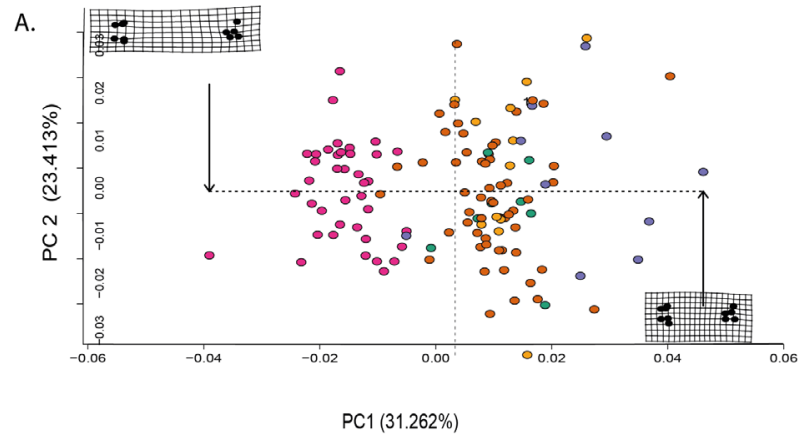

c.
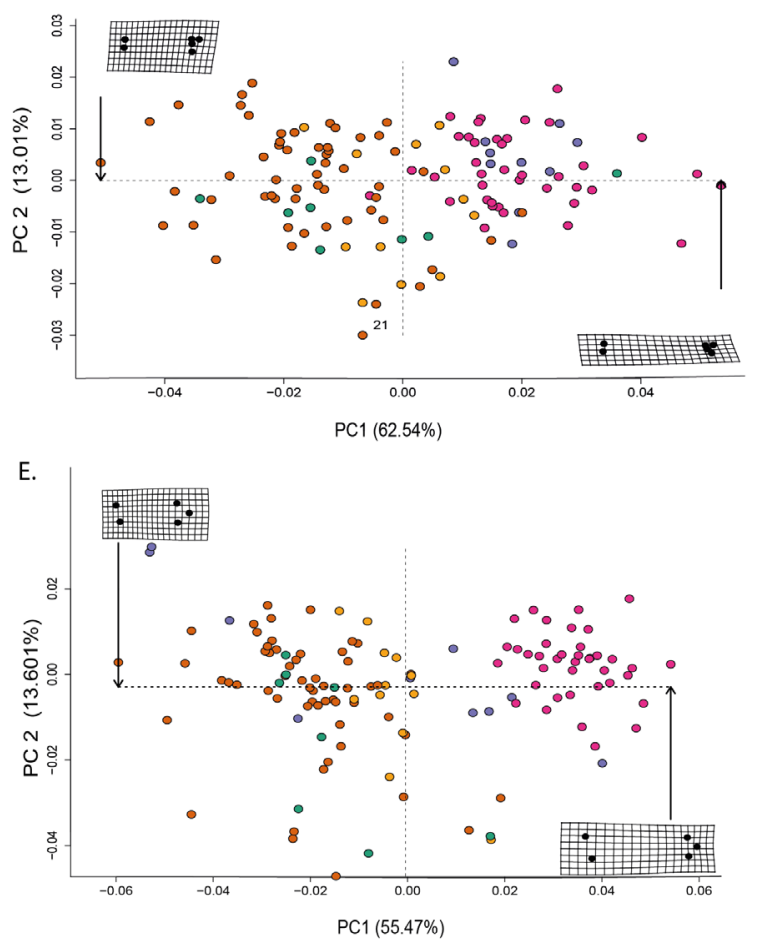

Figure 11.

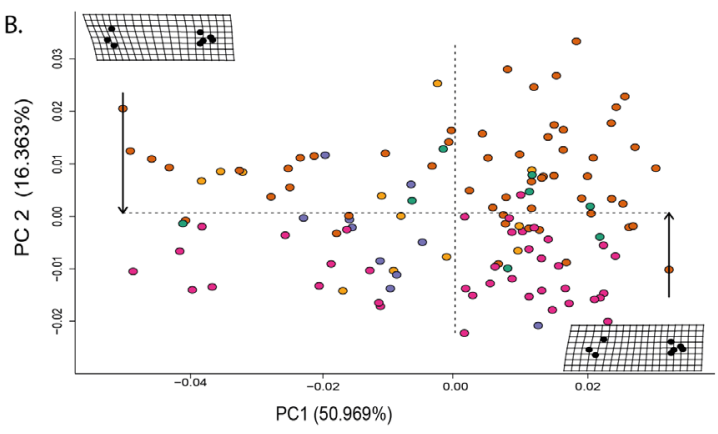

D.
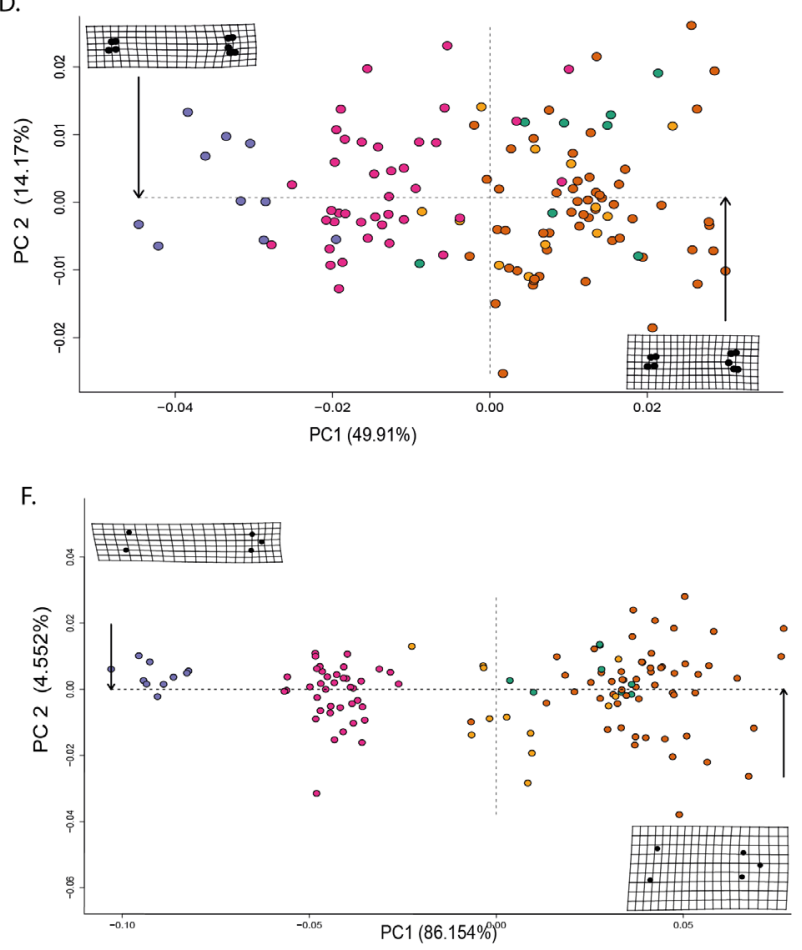

44 
Supporting information 
Figure S1: Landmark digitizing scheme for all bones. Number are landmark numbers - solid line indicates bone lenth measurement, dashed lines are additional measurements incorporated into the multipledistance index.

A.

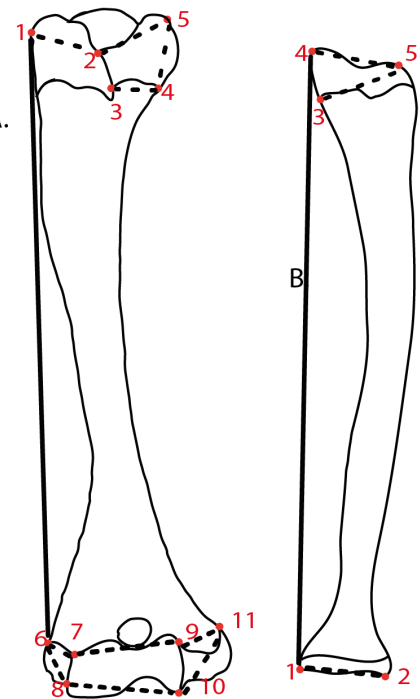

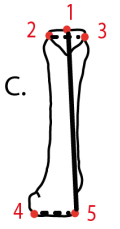
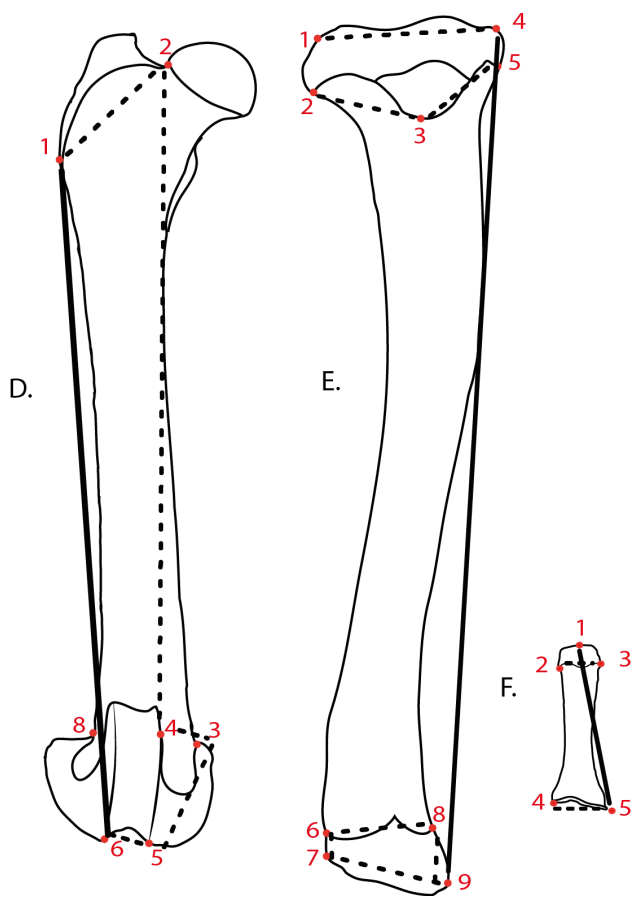
Figure S2: Results from the rarefaction of partial correlations (top panels) and edge exclusion deviance (EED)-values (bottom panels) from the multiple-distance-based partial correlation analyses in Isoodon obesulus (left) and P. gunnii (right). Stippled lines indicate the correlations/EED-values from the full sample. 

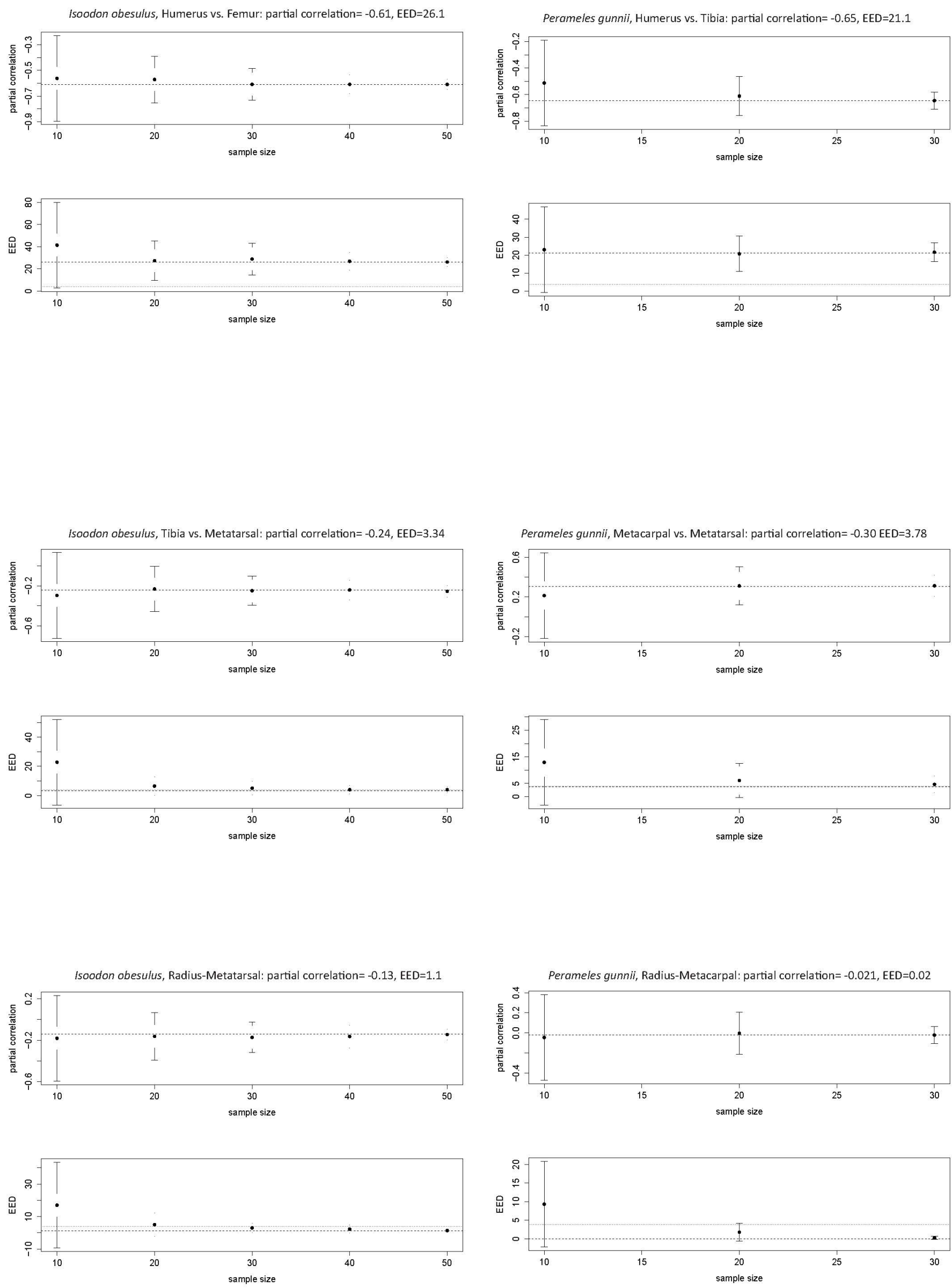
Figure S3: Results from the rarefaction of r-PLS (top panels) and p-values (bottom panels) from the 2B-PLS analysis of procustes shape in Isoodon obesulus (left) and P. gunnii (right). Stippled lines indicate the $r$ $\mathrm{PLS} / \mathrm{p}$-values from the full sample. 
Isoodon obesulus, Humerus vs. Femur: r-PLS: $0.773 ; p=0.002$
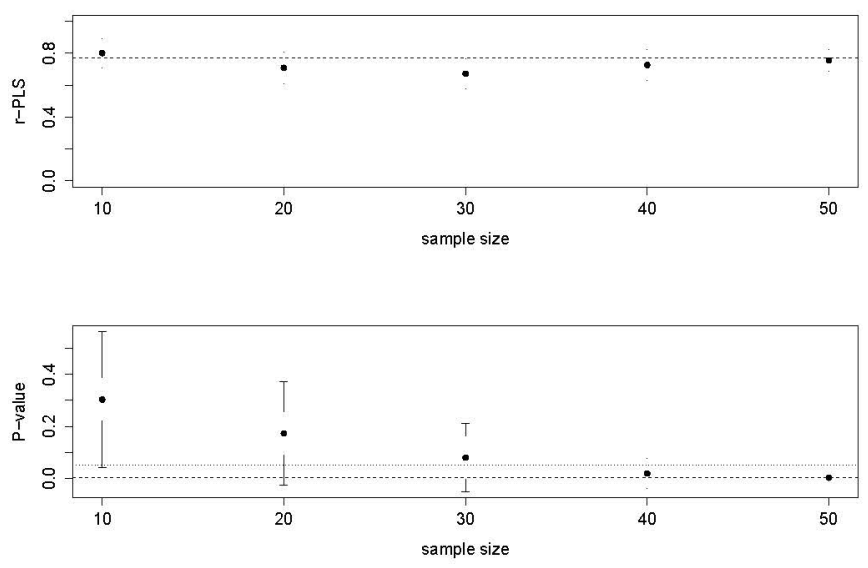

Isoodon obesulus, Femur vs. Radius: r-PLS: $0.41 ; \mathrm{p}=0.021$
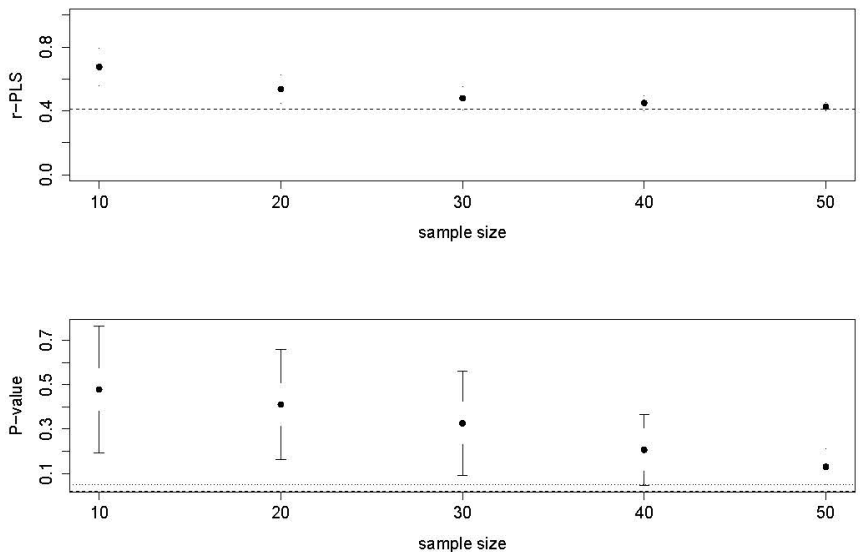

Isoodon obesulus, Radius vs. Metacarpal: r-PLS: $0.38 ; p=0.12$
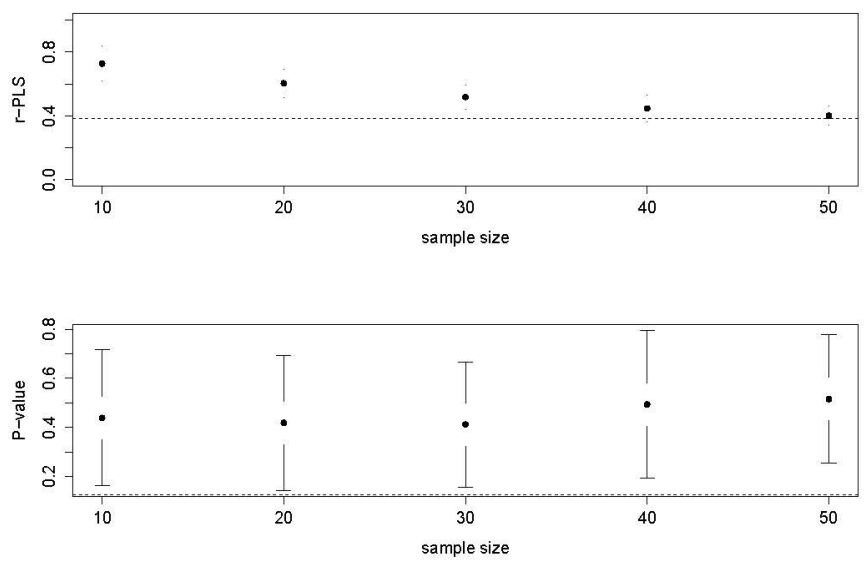

Perameles gunnii, Humerus vs. Metatarsal: r-PLS: 0.713; $p=0.002$
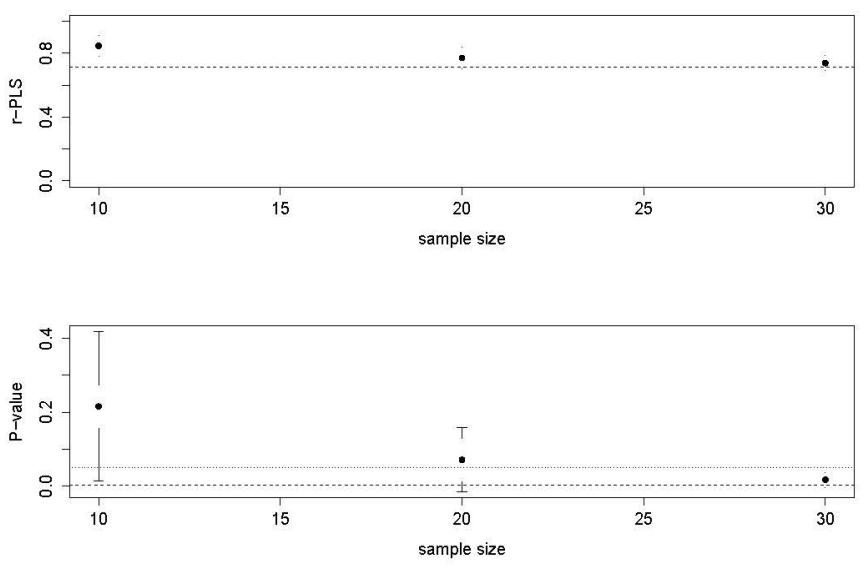

Perameles gunnii, Humerus vs. Radius: r-PLS: $0.44 ; p=0.108$
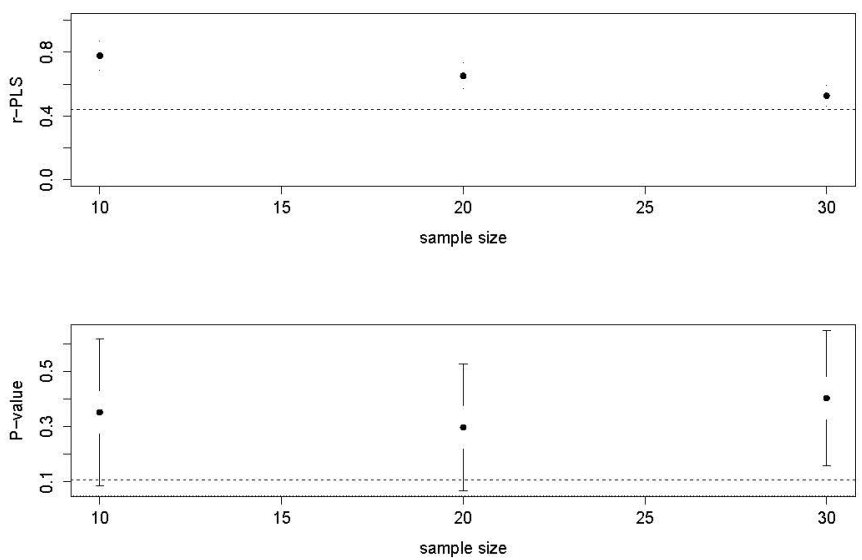

Perameles gunnii, Radius-Femur: r-PLS: 0.29; $p=0.226$
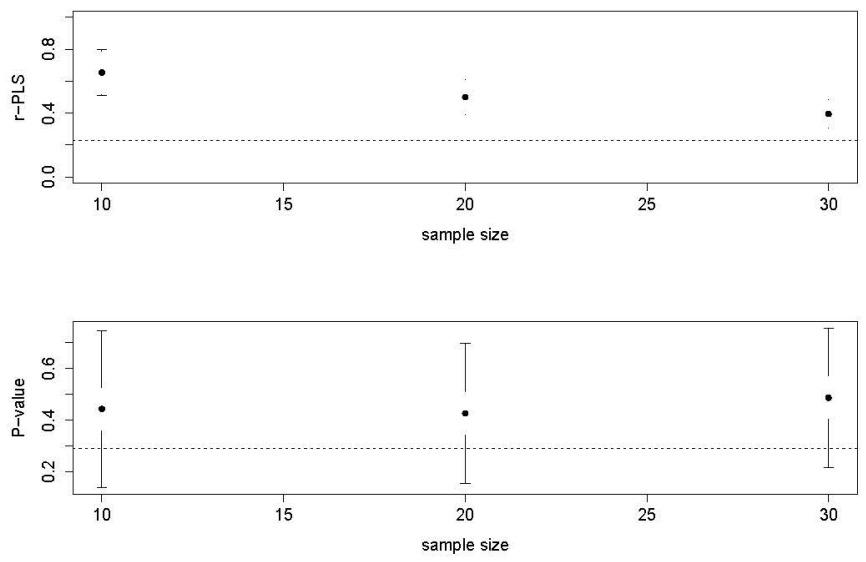
Fig.S4: Flow diagrams representing the limb bones that were significantly (solid lines) correlated in the ephiphyses-only based partial correlation matrices for each peramelemorph species. Dashed line is a representative of near significance. Bone abbreviations as in Fig. 1. See also Table S6 .

\section{I obesulus}
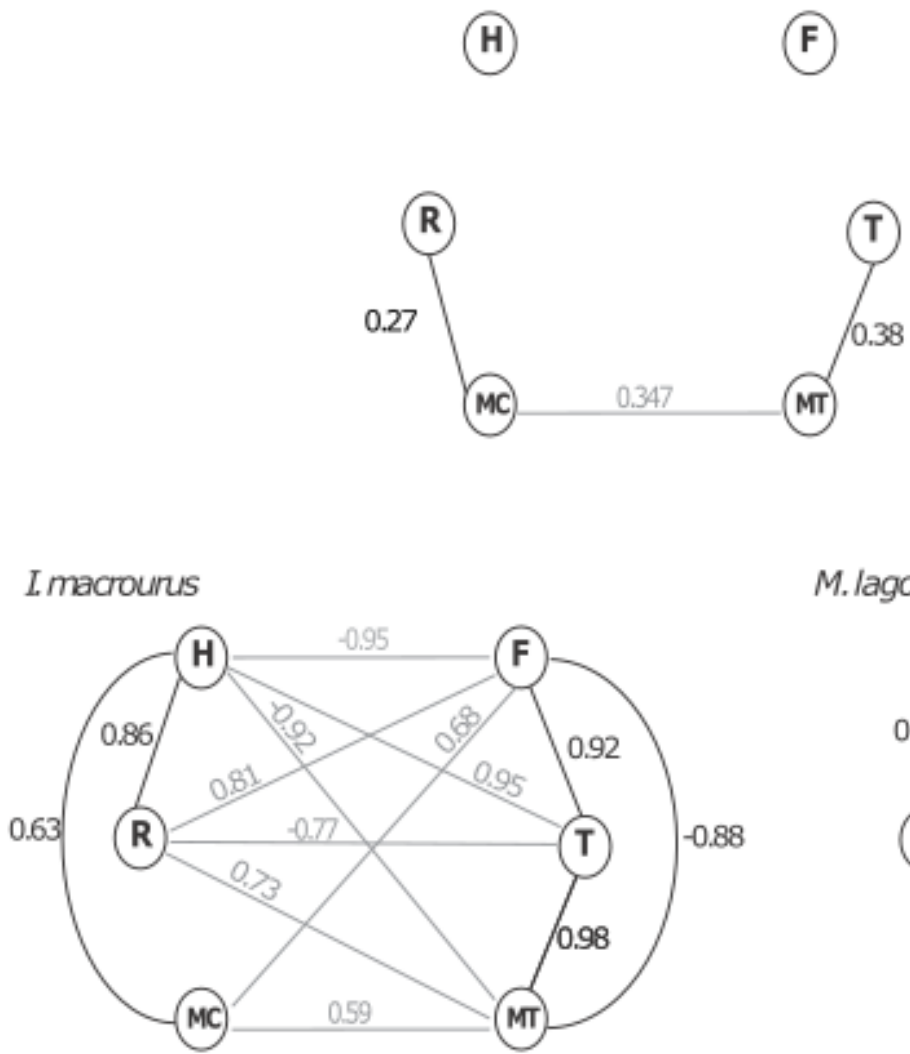

F

M. lagotis

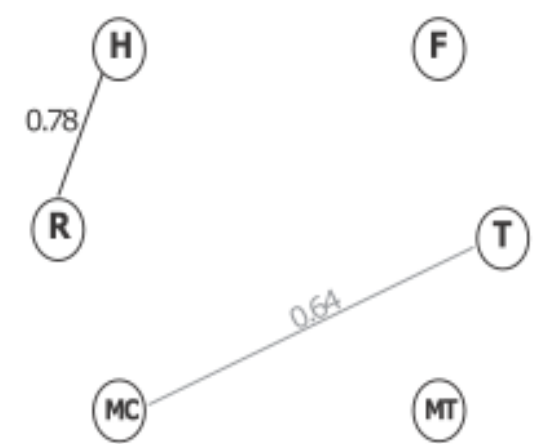

(F)

(R)

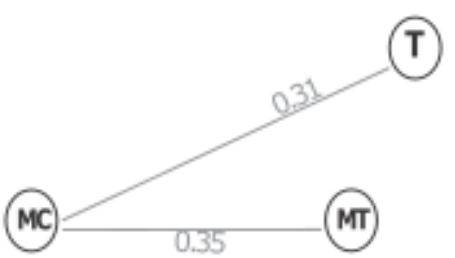


Table S1: Specimen list of each specimen used in final result analysis. The call number is the unique identification code used in the museum: WA = Western Australia, NSW= New South Wales, QLD= Queensland, TAS $=$ Tasmania, $\mathrm{SA}=$ South Australia, $\mathrm{NA}=$ not available. Species abbreviations: $1 \mathrm{O}=1$ soodon obesulus; IM=I. macrourus $\mathrm{PG}=$ Perameles gunnii $\mathrm{PN}=$ P.nasuta; $\mathrm{ML}=$ Macrotis lagotis.

\begin{tabular}{|c|c|c|c|c|c|c|c|c|c|c|c|}
\hline Call Number & $\begin{array}{l}\text { Specie } \\
\text { S }\end{array}$ & $\begin{array}{l}\text { Museu } \\
\mathrm{m}\end{array}$ & $\begin{array}{l}\text { Call } \\
\text { Number }\end{array}$ & $\begin{array}{l}\text { Speci } \\
\text { es }\end{array}$ & $\begin{array}{l}\text { Museu } \\
\text { m }\end{array}$ & Call Number & $\begin{array}{l}\text { Speci } \\
\text { es }\end{array}$ & $\begin{array}{l}\text { Museu } \\
\mathrm{m}\end{array}$ & $\begin{array}{l}\text { Call } \\
\text { Number }\end{array}$ & $\begin{array}{l}\text { Speci } \\
\text { es }\end{array}$ & $\begin{array}{l}\text { Museu } \\
\mathrm{m}\end{array}$ \\
\hline 19872 & IM & WA & 7257 & 10 & VIC & 45197 & 10 & WA & 32858 & PG & VIC \\
\hline 22082 & $\mathrm{IM}$ & WA & 7265 & 10 & SA & UZ30/ UZ13 & 10 & UWA & 32876 & PG & VIC \\
\hline M.35410 & IM & NSW & 7489 & 10 & WA & 14370 & $M L$ & WA & 32878 & PG & VIC \\
\hline M.35890 & IM & QLD & 7774 & 10 & VIC & 15935 & $M L$ & WA & 32879 & PG & VIC \\
\hline GIL101 & IM & NA & 9874 & 10 & WA & 16102 & $M L$ & WA & 32880 & PG & VIC \\
\hline M.36016 & IM & NSW & 10310 & 10 & VIC & 21,148 & ML & SA & 32881 & PG & VIC \\
\hline M.43499.002 & IM & NSW & 11953 & 10 & SA & M.22184.002 & ML & NSW & 32884 & PG & VIC \\
\hline JM807 & $\mathrm{IM}$ & QLD & 12174 & 10 & WA & 3,602 & ML & SA & 32885 & PG & VIC \\
\hline \#2 & 10 & WA & 12188 & 10 & WA & 3,606 & $M L$ & SA & 32891 & PG & VIC \\
\hline $\begin{array}{l}\text { QVM:2008:1:00 } \\
04\end{array}$ & 10 & TAS & 12194 & 10 & WA & M.37358.004 & ML & NSW & 32892 & PG & VIC \\
\hline $\begin{array}{l}\text { QVM:2014:1:00 } \\
34\end{array}$ & 10 & TAS & 12201 & 10 & WA & M.41236 & $M L$ & NSW & 32894 & PG & VIC \\
\hline $\begin{array}{l}\text { QVM:1976:1:01 } \\
07\end{array}$ & 10 & TAS & 13025 & 10 & SA & BM673 & $M L$ & SA & 32899 & PG & VIC \\
\hline $\begin{array}{l}\text { QVM:1976:1:01 } \\
12\end{array}$ & 10 & TAS & 13723 & 10 & WA & $\begin{array}{l}\text { QVM:1976:1:00 } \\
94\end{array}$ & PG & TAS & 32907 & PG & VIC \\
\hline $\begin{array}{l}\text { QVM:1976:1:01 } \\
15\end{array}$ & 10 & TAS & 18608 & 10 & VIC & 81.1.15 & PG & TAS & 32913 & PG & VIC \\
\hline $\begin{array}{l}\text { QVM:1988:1:00 } \\
85\end{array}$ & 10 & TAS & 18612 & 10 & VIC & 82.1 .15 & PG & TAS & 32917 & PG & VIC \\
\hline QVM:1995:1:2 & 10 & TAS & 18614 & 10 & VIC & $\begin{array}{l}\text { QVM:1983:1:00 } \\
51\end{array}$ & PG & TAS & 32974 & PG & VIC \\
\hline 5,231 & 10 & SA & 18954 & 10 & VIC & $\begin{array}{l}\text { QVM:1985:1:00 } \\
98\end{array}$ & PG & TAS & 32977 & PG & VIC \\
\hline 5735 & 10 & VIC & 19073 & 10 & WA & $\begin{array}{l}\text { QVM:1986:1:00 } \\
36\end{array}$ & PG & TAS & 32981 & PG & VIC \\
\hline 6560 & 10 & WA & 23483 & 10 & VIC & 8188 & PG & VIC & 37102 & PG & VIC \\
\hline 6561 & 10 & WA & 26014 & 10 & VIC & 16590 & PG & SA & M. 37522 & PG & NSW \\
\hline 6736 & 10 & WA & 26030 & 10 & VIC & 18469 & PG & VIC & 18500 & PN & VIC \\
\hline 6739 & 10 & WA & 26208 & 10 & VIC & 18470 & PG & NSW & 18626 & PN & VIC \\
\hline 6770 & 10 & WA & 26318 & 10 & VIC & 18483 & PG & VIC & 26001 & PN & VIC \\
\hline 6932 & 10 & WA & 26596 & 10 & VIC & 18492 & PG & VIC & 26148 & PN & VIC \\
\hline 7157 & 10 & VIC & 26672 & 10 & VIC & 21584 & PG & VIC & 26680 & PN & VIC \\
\hline 7166 & 10 & & 26675 & 10 & VIC & 24061 & PG & WA & 26682 & PN & VIC \\
\hline 7168 & 10 & VIC & 26681 & 10 & VIC & 24069 & PG & VIC & 28720 & PN & VIC \\
\hline 7171 & 10 & VIC & 26683 & 10 & VIC & 25856 & PG & VIC & 33610 & PN & NSW \\
\hline 7172 & 10 & VIC & 27468 & 10 & VIC & 25999 & PG & VIC & 37960 & PN & NSW \\
\hline 33022 & 10 & VIC & 29688 & 10 & VIC & 26029 & PG & VIC & 38905 & PN & NSW \\
\hline \multirow[t]{2}{*}{33015} & 10 & VIC & 41239 & 10 & WA & 31206 & PG & VIC & 7772 & PN & VIC \\
\hline & & & & & & & & & JM8757 & PN & QLD \\
\hline
\end{tabular}


Table S2: Definition of landmark (LM) position and anatomical significance in relation to muscle attachment or joint movement in Peramelemorphian limb bones. Anatomical information sourced from (Williams et al. 1987; Warburton et al. 2013) . MC IV denotes fourth metacarpal and MT IV denotes fourth metatarsal.

\begin{tabular}{|c|c|c|c|}
\hline \multirow{12}{*}{$\begin{array}{l}\text { Hume } \\
\text { rus }\end{array}$} & & Description & Anatomical Significance \\
\hline & 1 & $\begin{array}{l}\text { Most proximal and lateral point of the greater humeral } \\
\text { tuberosity }\end{array}$ & $\begin{array}{l}\text { Point where musculus supinator attaches to scapula } \\
\text { for movement }\end{array}$ \\
\hline & 2 & $\begin{array}{l}\text { Point on the greater trochanter that intersects with the lesser } \\
\text { trochanter on the distal side }\end{array}$ & As above \\
\hline & 3 & $\begin{array}{l}\text { Most distal and medial the greater humeral tuberosity (lower), } \\
\text { on the epiphyseal boundary }\end{array}$ & As above \\
\hline & 4 & $\begin{array}{l}\text { Most distal and medial the greater humeral tuberosity (lower), } \\
\text { on the epiphyseal boundary }\end{array}$ & As above \\
\hline & 5 & $\begin{array}{l}\text { Most proximal and medial point of the lesser tubercle humeral } \\
\text { tuberosity }\end{array}$ & As above \\
\hline & 6 & Most lateral point and distal point on the lateral epicondyle & $\begin{array}{l}\text { Point of attachment for the extensor digitorum } \\
\text { muscle }\end{array}$ \\
\hline & 7 & Most lateral and proximal point on the capitulum & $\begin{array}{l}\text { Area of joint movement for humerus, radius and } \\
\text { ulna }\end{array}$ \\
\hline & 8 & Most lateral and distal point on the capitulum & As above \\
\hline & 9 & Most medial and proximal point on the capitulum & As above \\
\hline & $\begin{array}{l}1 \\
0\end{array}$ & Most medial and distal point on the capitulum & As above \\
\hline & $\begin{array}{l}1 \\
1\end{array}$ & Most distal and medial point of the medial epicondyle & $\begin{array}{l}\text { Point where pronator teres muscle attaches - } \\
\text { involved in forearm rotation }\end{array}$ \\
\hline \multirow[t]{6}{*}{$\mathbf{R}$} & 1 & Most proximal and medial point of the head & $\begin{array}{l}\text { Point of movement and attachment to ulna and } \\
\text { humerus }\end{array}$ \\
\hline & 2 & Most proximal and medial point of the head & As above \\
\hline & 3 & Most medial and proximal point of the styloid process & $\begin{array}{l}\text { Point of movement and attachment to the } \\
\text { triquetrum and scaphoid }\end{array}$ \\
\hline & 4 & Most distal and medial point of the styloid process & As above \\
\hline & 5 & Most proximal and centroid point of the styloid process & As above \\
\hline & 6 & Most lateral and distal point of the styloid process & As above \\
\hline \multirow[t]{5}{*}{ MCIV } & 1 & Most proximal and lateral point of metacarpal head (upper) & $\begin{array}{l}\text { Point of attachment for the extensor digitorum } \\
\text { communis- involved in the extension of the } \\
\text { phalanges and joint movement of the forearm }\end{array}$ \\
\hline & 2 & Most proximal and medial point of metacarpal head (upper) & As above \\
\hline & 3 & Most distal and lateral side of metacarpal head & As above \\
\hline & 4 & Most distal and lateral side of the metacarpal base & $\begin{array}{l}\text { Point of attachment for adductor pollicis oblique } \\
\text { muscle }\end{array}$ \\
\hline & 5 & Most distal and medial point of the metacarpal base & $\begin{array}{l}\text { Point of attachment for extensor carpi radialis } \\
\text { muscle- involved in movement of the wrist }\end{array}$ \\
\hline \multirow[t]{8}{*}{ Femur } & 1 & Most proximal and lateral point of greater trochanter & $\begin{array}{l}\text { Point of attachment for the gluteus medius - } \\
\text { involved in the movement of the hip joint }\end{array}$ \\
\hline & 2 & Most proximal and medial point of the head (upper) & $\begin{array}{l}\text { Point of attachment for the synovial membrane - } \\
\text { involved in the movement of ball and socket joint } \\
\text { for femur extension }\end{array}$ \\
\hline & 3 & Most distal and lateral point on the head on the epiphyseal line & As above \\
\hline & 4 & Most distal and medial point on the medial epicondyle & Point of attachment for the synovial membrane \\
\hline & 5 & Most proximal point of the patella groove & $\begin{array}{l}\text { Point of attachment for the anterior cruciate } \\
\text { ligament - involved in forelimb extension }\end{array}$ \\
\hline & 6 & Most distal and medial point of the patella femoral groove & As above \\
\hline & 7 & Most distal and medial point on the patella groove & $\begin{array}{l}\text { Point of attachment for the popliteus - involved in } \\
\text { the flexion of the knee and rotation of the tibia }\end{array}$ \\
\hline & 8 & Most distal and lateral point of the patella femoral groove & $\begin{array}{l}\text { Point of attachment of the gastrocnemius lateral } \\
\text { muscle - involved in the propulsive movement of } \\
\text { the forelimb }\end{array}$ \\
\hline Tibia & 1 & Most proximal and lateral point on the lateral condyle & $\begin{array}{l}\text { Point of attachment for extensor digitorum longus } \\
\text { that connects to the fibula - involved in extension }\end{array}$ \\
\hline
\end{tabular}


2 Most distal and lateral point on the lateral condyle, on the epiphyseal boundary

3 Most distal and central point of the tibia tuberosity

4 Most proximal and medial point of the medial condyle

5 Most distal an medial point of the medial condyle

6 Most proximal and lateral point of the lateral malleolus, on the epiphyseal boundary

7 Most distal and lateral point of the lateral malleolus

8 Most proximal and medial point of medial malleolus

9 Most distal and distal point of the medial condyle

MT IV 1 Most proximal and lateral point of metatarsal head (upper)

2 Most proximal and medial point of metatarsal head (upper)

3 Most distal and lateral side of metatarsal head

4 Most distal and lateral side of the metatarsal base

5 Most distal and medial point of the metatarsal base
As above

Point of attachment for the tendon of quadriceps femoris that connects to the patella - involved in extension of the femur

Point of attachment for the extensor digitorum longus II and III - involved in the extension the phalanges

As above

As above

As above

As above

As above

Point of attachment for the tibialis anterior muscle

- involved in the extension of the phalanges

As above

As above

As above

As above 
Table S3: Variance/Covariance matrix repeatability results generated from random skewers analysis. $n=$ number of specimens in the sample.

\begin{tabular}{llll}
\hline Datasets & Species & $\mathbf{n}$ & Repeatability \\
\hline \multirow{4}{*}{ Length-only } & I. obesulus & 56 & 0.977 \\
& P. gunnii & 29 & 0.972 \\
& P. nasuta & 12 & 0.915 \\
& M. lagotis & 10 & 0.883 \\
& I. macrourus & 8 & 0.913 \\
\hline \multirow{4}{*}{ Multiple-distance } & I. obesulus & 56 & 0.975 \\
& P. gunnii & 29 & 0.965 \\
& P. nasuta & 12 & 0.917 \\
& M. lagotis & 10 & 0.892 \\
& I. macrourus & 8 & 0.882 \\
\hline
\end{tabular}


Table S4: Results from the population structure analyses for geometric morphometric (GM) and linear measurement (LM) datasets assessing the contribution of size (centroid size for GM/geometric mean for LM datasets), age (adult vs. subadult), location ( 4 in for $I$. obesulus, 5 for $P$. nasuta), sex, and left vs. right side on shape variation. 


\begin{tabular}{|c|c|c|c|c|c|c|c|c|c|c|c|c|c|c|}
\hline \multirow{8}{*}{ Humerus } & \multicolumn{8}{|c|}{ Isoodon obesulus, $\mathrm{n}=42$} & \multicolumn{6}{|c|}{ Perameles gunnii, $n=23$} \\
\hline & \multirow[t]{2}{*}{ Factor } & \multicolumn{4}{|c|}{ GM } & \multicolumn{3}{|c|}{ LM } & \multicolumn{3}{|c|}{ GM } & \multicolumn{3}{|c|}{ LM } \\
\hline & & Df & Rsq & $\mathbf{F}$ & $p$ & Rsq & $\mathbf{F}$ & $p$ & Rsq & $\mathbf{F}$ & $p$ & Rsq & $\mathbf{F}$ & $p$ \\
\hline & Size & 1 & 0.19 & 9.40 & 0.001 & 0.97 & 1358.44 & 0.001 & 0.03 & 0.75 & 0.802 & 0.83 & 90.46 & 0.001 \\
\hline & Sex & 1 & 0.038 & 1.91 & 0.052 & 0.00 & 0.50 & 0.662 & 0.05 & 1.28 & 0.368 & 0.02 & 1.97 & 0.120 \\
\hline & Location & $4 / 5$ & 0.12 & 1.19 & 0.135 & 0.01 & 2.92 & 0.009 & 0.31 & 1.64 & 0.042 & 0.02 & 0.49 & 0.759 \\
\hline & Age & 1 & 0.02 & 0.80 & 0.441 & 0.00 & 1.95 & 0.097 & 0.04 & 1.03 & 0.185 & 0.01 & 0.57 & 0.393 \\
\hline & Side & 1 & 0.01 & 0.62 & 0.667 & 0.00 & 0.86 & 0.315 & 0.08 & 2.07 & 0.005 & 0.01 & 1.05 & 0.196 \\
\hline \multirow[t]{5}{*}{ Radius } & Size & 1 & 0.26 & 15.74 & 0.001 & 0.97 & 1661.74 & 0.001 & 0.05 & 1.29 & 0.299 & 0.88 & 161.08 & 0.001 \\
\hline & Sex & 1 & 0.01 & 0.40 & 0.869 & 0.00 & 0.07 & 0.992 & 0.02 & 0.41 & 0.797 & 0.01 & 1.06 & 0.329 \\
\hline & Location & $4 / 5$ & 0.10 & 1.15 & 0.380 & 0.00 & 2.09 & 0.095 & 0.33 & 1.62 & 0.083 & 0.03 & 1.55 & 0.180 \\
\hline & Age & 1 & 0.02 & 1.46 & 0.164 & 0.00 & 4.25 & 0.019 & 0.01 & 0.32 & 0.713 & 0.00 & 0.11 & 0.893 \\
\hline & Side & 1 & 0.09 & 5.31 & 0.001 & 0.00 & 0.24 & 0.769 & 0.07 & 1.69 & 0.066 & 0.01 & 1.92 & 0.082 \\
\hline \multirow[t]{5}{*}{ MC } & Size & 1 & 0.05 & 1.97 & 0.108 & 0.83 & 172.36 & 0.001 & 0.13 & 4.16 & 0.027 & 0.64 & 31.72 & 0.001 \\
\hline & Sex & 1 & 0.02 & 0.82 & 0.484 & 0.00 & 0.32 & 0.652 & 0.12 & 3.83 & 0.027 & 0.01 & 0.26 & 0.653 \\
\hline & Location & $4 / 5$ & 0.12 & 1.00 & 0.368 & 0.01 & 0.53 & 0.717 & 0.33 & 2.20 & 0.009 & 0.06 & 0.76 & 0.490 \\
\hline & Age & 1 & 0.02 & 0.87 & 0.382 & 0.01 & 1.41 & 0.189 & 0.02 & 0.64 & 0.404 & 0.01 & 0.57 & 0.339 \\
\hline & Side & 1 & 0.02 & 0.88 & 0.351 & 0.00 & 0.20 & 0.746 & 0.02 & 0.61 & 0.418 & 0.02 & 1.15 & 0.179 \\
\hline \multirow[t]{5}{*}{ Femur } & Size & 1 & 0.09 & 3.74 & 0.018 & 0.95 & 720.54 & 0.001 & 0.01 & 0.10 & 0.988 & 0.76 & 60.99 & 0.001 \\
\hline & Sex & 1 & 0.01 & 0.45 & 0.732 & 0.00 & 0.36 & 0.731 & 0.02 & 0.38 & 0.788 & 0.00 & 0.31 & 0.752 \\
\hline & Location & $4 / 5$ & 0.08 & 0.83 & 0.516 & 0.00 & 0.91 & 0.447 & 0.28 & 1.10 & 0.281 & 0.05 & 1.07 & 0.267 \\
\hline & Age & 1 & 0.01 & 0.39 & 0.701 & 0.00 & 0.64 & 0.484 & 0.04 & 0.74 & 0.299 & 0.01 & 0.41 & 0.539 \\
\hline & Side & 1 & 0.03 & 1.40 & 0.146 & 0.00 & 2.60 & 0.050 & 0.06 & 1.10 & 0.139 & 0.01 & 0.86 & 0.274 \\
\hline \multirow[t]{5}{*}{ Tibia } & Size & 1 & 0.14 & 7.07 & 0.001 & 0.97 & 1697.22 & 0.001 & 0.09 & 3.22 & 0.062 & 0.74 & 69.30 & 0.001 \\
\hline & Sex & 1 & 0.04 & 1.89 & 0.091 & 0.00 & 0.34 & 0.826 & 0.09 & 3.10 & 0.059 & 0.01 & 0.96 & 0.382 \\
\hline & Location & 4 & 0.16 & 1.63 & 0.031 & 0.01 & 2.66 & 0.021 & 0.41 & 2.86 & 0.001 & 0.10 & 2.39 & 0.035 \\
\hline & Age & 1 & 0.02 & 1.03 & 0.273 & 0.00 & 0.45 & 0.582 & 0.02 & 0.55 & 0.575 & 0.00 & 0.10 & 0.905 \\
\hline & Side & 1 & 0.03 & 1.76 & 0.031 & 0.00 & 2.73 & 0.044 & 0.03 & 1.04 & 0.105 & 0.01 & 0.54 & 0.386 \\
\hline \multirow[t]{5}{*}{ MT } & Size & 1 & 0.07 & 3.72 & 0.013 & 0.84 & 245.50 & 0.001 & 0.31 & 16.99 & 0.019 & 0.78 & 60.95 & 0.001 \\
\hline & Sex & 1 & 0.02 & 0.78 & 0.611 & 0.00 & 0.33 & 0.701 & 0.14 & 7.71 & 0.010 & 0.01 & 0.45 & 0.552 \\
\hline & Location & 4 & 0.20 & 1.98 & 0.026 & 0.04 & 3.09 & 0.014 & 0.30 & 3.28 & 0.002 & 0.03 & 0.54 & 0.760 \\
\hline & Age & 1 & 0.02 & 1.2294 & 0.212 & 0.00 & 0.21 & 0.729 & 0.03 & 1.45 & 0.081 & 0.01 & 0.41 & 0.499 \\
\hline & Side & 1 & 0.02 & 0.85 & 0.381 & 0.00 & 0.42 & 0.521 & 0.01 & 0.62 & 0.389 & 0.01 & 0.76 & 0.280 \\
\hline
\end{tabular}


Table S5: Results from the population structure analyses of geometric morphometric (GM) and linear measurement (LM) datasets whether slopes between adult and subadult specimens were different, and whether the distinction resulted in significant size differences, in all specimens for which size data were available. Note that with the larger sample sizes, there is no significant difference between differently aged individuals, with one low-significance instance of slope differences in the linear measurements.

\begin{tabular}{|c|c|c|c|c|c|c|c|c|c|c|c|c|c|}
\hline & \multicolumn{7}{|c|}{ Isoodon obesulus, $\mathrm{n}=48$} & \multicolumn{6}{|c|}{ Perameles gunnii, $\mathrm{n}=29$} \\
\hline & \multicolumn{4}{|c|}{ GM } & \multicolumn{3}{|c|}{ LM } & \multicolumn{3}{|c|}{$\overline{G M}$} & \multicolumn{3}{|c|}{ LM } \\
\hline & & Rsq & $\mathbf{F}$ & $p$ & Rsq & $\mathbf{F}$ & $p$ & Rsq & $\mathbf{F}$ & $p$ & Rsq & $\mathbf{F}$ & $p$ \\
\hline \multirow[t]{3}{*}{ Humerus } & Size & 0.97 & 1336.22 & 0.001 & 0.97 & 1267.36 & 0.001 & 0.81 & 103.29 & 0.001 & 0.82 & 125.14 & 0.001 \\
\hline & Age & 0.00 & 0.89 & 0.333 & 0.00 & 1.24 & 0.281 & 0.00 & 0.62 & 0.486 & 0.01 & 0.76 & 0.414 \\
\hline & Size:Age & 0.00 & 0.79 & 0.463 & 0.00 & 0.35 & 0.741 & 1.04 & 0.91 & 0.343 & 0.01 & 1.18 & 0.267 \\
\hline \multirow[t]{3}{*}{ Radius } & Size & 0.91 & 463.76 & 0.001 & 0.97 & 1758.67 & 0.001 & 0.88 & 177.86 & 0.001 & 0.88 & 13.06 & 0.001 \\
\hline & Age & 0.00 & 0.18 & 0.770 & 0.00 & 2.42 & 0.134 & 0.00 & 0.49 & 0.550 & 0.00 & 0.40 & 0.487 \\
\hline & Size:Age & 0.01 & 1.78 & 0.154 & 0.00 & 4.42 & 0.024 & 0.01 & 0.75 & 0.484 & 0.01 & 1.19 & 0.166 \\
\hline \multirow[t]{3}{*}{ Metacarpal } & Size & 0.72 & 114.64 & 0.001 & 0.82 & 215.54 & 0.001 & 0.70 & 55.29 & 0.001 & 0.63 & 44.70 & 0.001 \\
\hline & Age & 0.00 & 0.05 & 0.973 & 0.00 & 0.13 & 0.912 & 0.00 & 0.15 & 0.821 & 0.00 & 0.06 & 0.974 \\
\hline & Size:Age & 0.00 & 0.22 & 0.835 & 0.01 & 2.10 & 0.126 & 0.01 & 0.40 & 0.655 & 0.02 & 1.53 & 0.176 \\
\hline \multirow[t]{3}{*}{ Femur } & Size & 0.95 & 798.85 & 0.001 & 0.94 & 766.75 & 0.001 & 0.75 & 74.75 & 0.001 & 0.76 & 81.46 & 0.001 \\
\hline & Age & 0.00 & 0.27 & 0.843 & 0.00 & 0.26 & 0.806 & 0.00 & 0.38 & 0.730 & 0.00 & 0.42 & 0.675 \\
\hline & Size:Age & 0.00 & 1.20 & 0.308 & 0.00 & 0.81 & 0.404 & 0.01 & 0.46 & 0.780 & 0.01 & 0.68 & 0.502 \\
\hline \multirow[t]{3}{*}{ Tibia } & Size & 0.95 & 938.26 & 0.001 & 0.97 & 1799.66 & 0.001 & 0.75 & 75.42 & 0.001 & 0.75 & 86.92 & 0.001 \\
\hline & Age & 0.00 & 0.35 & 0.624 & 0.00 & 0.65 & 0.516 & 0.01 & 0.87 & 0.347 & 0.01 & 1.01 & 0.328 \\
\hline & Size:Age & 0.00 & 1.70 & 0.153 & 0.00 & 1.67 & 0.148 & 0.03 & 1.87 & 0.127 & 0.03 & 3.20 & 0.061 \\
\hline \multirow[t]{3}{*}{ Metatarsal } & Size & 0.67 & 89.78 & 0.001 & 0.85 & 256.48 & 0.001 & 0.77 & 84.37 & 0.001 & 0.76 & 92.87 & 0.001 \\
\hline & Age & 0.00 & 0.02 & 0.985 & 0.00 & 0.46 & 0.552 & 0.01 & 0.68 & 0.448 & 0.00 & 0.50 & 0.613 \\
\hline & Size:Age & 0.00 & 0.10 & 0.939 & 0.01 & 1.79 & 0.142 & 0.03 & 1.73 & 0.145 & 0.03 & 3.98 & 0.038 \\
\hline
\end{tabular}


Table S6: Length-only partial correlation matrices for each Peramelemorphian species. Partial correlation values are in the lower triangle, and associated edge exclusion values (EED) are in the upper triangle. Bold indicates significant pairwise tests (EED $\geq 3.84$ ). Grey cells are within-bone correlations and boxed cells are serially homologous limb bone correlations. MC = Metacarpal and MT= Metatarsal.

\begin{tabular}{|c|c|c|c|c|c|c|c|c|c|c|c|c|c|}
\hline & Humerus & Radius & MC & Femur & Tibia & MT & & Humerus & Radius & MC & Femur & Tibia & MT \\
\hline I. obesulus & & & & & & & P. gunnii & & & & & & \\
\hline Humerus & & 0.872 & 0.213 & 8.530 & 0.383 & 1.813 & & & 0.265 & 0.518 & 0.288 & 0.300 & 0.247 \\
\hline Radius & -0.124 & & 0.096 & 1.879 & 11.544 & 1.416 & & -0.082 & & 0.273 & 0.005 & 10.045 & 0.953 \\
\hline MC & -0.062 & -0.041 & & 4.215 & 8.712 & 26.907 & & -0.115 & 0.084 & & 0.367 & 2.307 & 10.338 \\
\hline Femur & 0.376 & 0.182 & 0.269 & & 3.522 & 0.183 & & -0.086 & -0.011 & -0.097 & & 6.598 & 0.179 \\
\hline Tibia & -0.083 & 0.432 & -0.380 & 0.247 & & 2.920 & & 0.087 & 0.477 & -0.240 & 0.395 & & 13.847 \\
\hline MT & -0.178 & -0.158 & 0.618 & -0.057 & 0.225 & & & -0.080 & -0.155 & 0.483 & 0.068 & 0.547 & \\
\hline I. macrourus & & & & & & & P. nasuta & & & & & & \\
\hline Humerus & & 0.112 & 0.003 & 3.725 & 0.104 & 0.208 & & & 1.405 & 0.851 & 2.029 & 4.112 & 0.014 \\
\hline Radius & 0.118 & & 7.974 & 2.758 & 12.250 & 6.004 & & -0.332 & & 0.644 & 0.344 & 2.939 & 1.248 \\
\hline MC & 0.020 & 0.794 & & 3.339 & 18.016 & 14.181 & & -0.262 & -0.229 & & 0.599 & 0.323 & 12.630 \\
\hline Femur & 0.610 & -0.540 & 0.584 & & 5.876 & 1.204 & & 0.394 & 0.168 & 0.221 & & 0.960 & 1.642 \\
\hline Tibia & -0.114 & 0.885 & -0.946 & 0.721 & & 9.712 & & 0.539 & 0.466 & -0.163 & 0.277 & & 1.638 \\
\hline MT & -0.160 & -0.727 & 0.911 & -0.374 & 0.838 & & & 0.034 & 0.314 & 0.807 & -0.358 & 0.357 & \\
\hline \multicolumn{14}{|l|}{ M. lagotis } \\
\hline Humerus & & 0.003 & 0.714 & 0.062 & 1.944 & 2.519 & & & & & & & \\
\hline Radius & 0.016 & & 0.625 & 5.963 & 2.069 & 0.000 & & & & & & & \\
\hline MC & 0.263 & 0.246 & & 0.098 & 5.239 & 10.250 & & & & & & & \\
\hline Femur & 0.078 & 0.670 & -0.099 & & 0.212 & 0.188 & & & & & & & \\
\hline Tibia & 0.420 & 0.432 & -0.639 & -0.145 & & 4.300 & & & & & & & \\
\hline MT & -0.472 & -0.006 & 0.801 & -0.136 & 0.591 & & & & & & & & \\
\hline
\end{tabular}


Table S7: Multiple-distance partial correlation matrix for each peramelemorph species. Partial correlation values are in the lower triangle, and associated Edge exclusion values (EED) are in the upper triangle. Bold indicates significant pairwise tests (EED $\geq 3.84$ ). Grey cells are within-bone correlations and boxed cells are serially homologous limb bone correlations. MC = Metacarpal and MT= Metatarsal.

\begin{tabular}{|c|c|c|c|c|c|c|c|c|c|c|c|c|}
\hline & Humerus & Radius & MC & Femur & Tibia & MT & Humerus & Radius & MC & Femur & Tibia & MT \\
\hline I. obesulus & & & & & & & & & & & & \\
\hline Humerus & & 7.579 & 3.130 & 26.082 & 42.242 & 13.272 & & 7.299 & 5.915 & 2.692 & 21.055 & 0.214 \\
\hline Radius & -0.356 & & 2.122 & 6.311 & 1.957 & 1.089 & -0.413 & & 0.018 & 0.741 & 7.883 & 0.528 \\
\hline MC & -0.233 & -0.193 & & 4.576 & 7.229 & 11.569 & -0.375 & -0.021 & & 12.288 & 0.713 & 3.777 \\
\hline Femur & -0.610 & -0.326 & -0.280 & & 16.512 & 7.308 & -0.258 & 0.137 & -0.520 & & 1.483 & 0.061 \\
\hline Tibia & -0.728 & -0.185 & -0.348 & -0.505 & & 3.344 & -0.646 & -0.428 & -0.135 & 0.193 & & 1.127 \\
\hline MT & -0.459 & -0.139 & 0.432 & -0.350 & -0.241 & & -0.074 & 0.116 & 0.304 & 0.040 & 0.169 & \\
\hline $\begin{array}{l}\text { I. } \\
\text { macrourus }\end{array}$ & & & & & & & & & & & & \\
\hline Humerus & & 0.000 & 0.219 & 0.022 & 0.488 & 2.969 & & 0.549 & 4.611 & 0.536 & 0.386 & 2.788 \\
\hline Radius & 0.003 & & 2.312 & 4.483 & 0.651 & 1.964 & -0.211 & & 0.956 & 0.431 & 1.840 & 1.531 \\
\hline MC & 0.164 & -0.501 & & 0.904 & 2.973 & 0.455 & -0.565 & -0.277 & & 0.241 & 1.796 & 20.181 \\
\hline Femur & -0.052 & -0.655 & -0.327 & & 0.083 & 0.680 & 0.209 & 0.188 & 0.141 & & 1.501 & 1.587 \\
\hline Tibia & 0.243 & 0.280 & 0.557 & -0.101 & & 0.380 & -0.178 & -0.377 & -0.373 & -0.343 & & 1.179 \\
\hline MT & -0.557 & -0.467 & 0.235 & -0.285 & 0.215 & & 0.455 & 0.346 & 0.902 & -0.352 & 0.306 & \\
\hline \multicolumn{13}{|l|}{ M. lagotis } \\
\hline Humerus & & 3.415 & 2.374 & 0.006 & 5.966 & 0.045 & & & & & & \\
\hline Radius & -0.538 & & 4.923 & 5.900 & 0.297 & 3.833 & & & & & & \\
\hline MC & -0.460 & -0.624 & & 1.767 & 1.063 & 11.010 & & & & & & \\
\hline Femur & -0.024 & 0.668 & 0.402 & & 2.042 & 4.284 & & & & & & \\
\hline Tibia & -0.670 & -0.171 & -0.318 & -0.430 & & 0.009 & & & & & & \\
\hline MT & 0.067 & 0.564 & 0.817 & -0.590 & 0.031 & & & & & & & \\
\hline
\end{tabular}


Table S8: Associated multiple-distance biplot Principal Component 1 (PC1) Principal Component 2 (PC2) eigenvalues. $R^{2}$ denotes $r$-squared values and Pr(>r) denotes p-values. $\mathrm{MC}=$ Metacarpal and MT= Metatarsal.

\begin{tabular}{llllll}
\hline Species & Limb bone vector & $\mathbf{P C 1}$ & $\mathbf{P C 2}$ & $\mathbf{R}^{\mathbf{2}}$ & $\mathbf{P r}(>\mathbf{r})$ \\
\hline I. obesulus & Humerus & 0.624 & -0.782 & 0.83 & $\mathbf{0 . 0 0 0}$ \\
& Radius & -0.254 & 0.967 & 0.22 & $\mathbf{0 . 0 0 1}$ \\
& MC & -0.825 & -0.565 & 0.80 & $\mathbf{0 . 0 0 0}$ \\
& Femur & 0.753 & 0.658 & 0.24 & $\mathbf{0 . 0 0 1}$ \\
& Tibia & -0.294 & 0.956 & 0.70 & $\mathbf{0 . 0 0 0}$ \\
& MT & -0.968 & -0.251 & 0.82 & $\mathbf{0 . 0 0 0}$ \\
\hline P. gunnii & Humerus & 0.999 & -0.043 & 0.82 & $\mathbf{0 . 0 0 0}$ \\
& Radius & -0.879 & 0.477 & 0.15 & $\mathbf{0 . 0 5 0}$ \\
& MC & -0.229 & 0.973 & 0.81 & $\mathbf{0 . 0 0 0}$ \\
& Femur & -0.531 & -0.847 & 0.80 & $\mathbf{0 . 0 0 0}$ \\
& Tibia & -0.934 & -0.357 & 0.70 & $\mathbf{0 . 0 0 0}$ \\
& MT & -0.758 & 0.652 & 0.59 & $\mathbf{0 . 0 0 0}$ \\
\hline I. macrourus & Humerus & 0.453 & 0.892 & 0.58 & 0.117 \\
& Radius & 0.683 & 0.730 & 0.82 & $\mathbf{0 . 0 2 0}$ \\
& MC & -0.961 & 0.278 & 0.88 & $\mathbf{0 . 0 0 5}$ \\
& Femur & 0.282 & -0.959 & 0.74 & $\mathbf{0 . 0 4 1}$ \\
& Tibia & -0.685 & 0.729 & 0.84 & $\mathbf{0 . 0 1 0}$ \\
& MT & -0.969 & -0.248 & 0.89 & $\mathbf{0 . 0 0 6}$ \\
\hline P. nasuta & Humerus & 0.907 & 0.420 & 0.37 & 0.129 \\
& Radius & 0.029 & -1.000 & 0.61 & $\mathbf{0 . 0 1 2}$ \\
& MC & -0.900 & -0.435 & 0.88 & $\mathbf{0 . 0 0 0}$ \\
& Femur & 0.824 & -0.567 & 0.73 & $\mathbf{0 . 0 0 3}$ \\
& Tibia & -0.318 & 0.948 & 0.74 & $\mathbf{0 . 0 0 2}$ \\
& MT & -0.961 & -0.278 & 0.84 & $\mathbf{0 . 0 0 0}$ \\
\hline Magotis & Humerus & 0.905 & 0.426 & 0.77 & $\mathbf{0 . 0 0 5}$ \\
& Radius & -0.120 & -0.993 & 0.78 & $\mathbf{0 . 0 0 7}$ \\
& MC & -0.955 & -0.297 & 0.66 & $\mathbf{0 . 0 2 7}$ \\
& Femur & 0.375 & -0.927 & 0.91 & $\mathbf{0 . 0 0 1}$ \\
& MT & -0.687 & 0.727 & 0.60 & $\mathbf{0 . 0 3 9}$ \\
& & -0.989 & -0.147 & 0.83 & $\mathbf{0 . 0 0 3}$ \\
\hline & & & & \\
& & & &
\end{tabular}


Table S9: Two-block partial least-squares (2B-PLS) results for each peramelemorphian species. PLS coefficients are in the lower triangle, and associated Pvalues are in the upper triangle. Bold indicates significant pairwise tests ( $p$-value $\leq 0.05$ ). Italicised indicates partially significant ( $p$-value $\leq 0.006$ ). Grey cells are within-bone correlations and boxed cells are serially homologous limb bone correlations. MC = Metacarpal and MT= Metatarsal.

\begin{tabular}{|c|c|c|c|c|c|c|c|c|c|c|c|c|c|}
\hline & Humerus & Radius & MC & Femur & Tibia & MT & & Humerus & Radius & MC & Femur & Tibia & MT \\
\hline \multicolumn{14}{|l|}{ I. obesulus } \\
\hline Humerus & & 0.002 & 0.027 & 0.000 & 0.086 & 0.000 & P. gunnii & & 0.124 & 0.002 & 0.130 & 0.001 & 0.000 \\
\hline Radius & 0.578 & & 0.130 & 0.018 & 0.530 & 0.008 & & 0.447 & & 0.023 & 0.234 & 0.002 & 0.020 \\
\hline $\mathrm{MC}$ & 0.505 & 0.383 & & 0.008 & 0.036 & 0.001 & & 0.656 & 0.497 & & 0.136 & 0.002 & 0.002 \\
\hline Femur & 0.773 & 0.413 & 0.481 & & 0.429 & 0.008 & & 0.423 & 0.292 & 0.357 & & 0.015 & 0.012 \\
\hline Tibia & 0.404 & 0.300 & 0.429 & 0.230 & & 0.178 & & 0.365 & 0.400 & 0.640 & 0.523 & & 0.005 \\
\hline MT & 0.635 & 0.503 & 0.618 & 0.618 & 0.379 & & & 0.714 & 0.512 & 0.636 & 0.501 & 0.621 & \\
\hline \multicolumn{7}{|l|}{ I. macrourus } & \multicolumn{7}{|l|}{ P. nasuta } \\
\hline Humerus & & 0.155 & 0.124 & 0.134 & 0.543 & 0.056 & & & 0.010 & 0.097 & 0.040 & 0.062 & 0.047 \\
\hline Radius & 0.740 & & 0.008 & 0.192 & 0.164 & 0.079 & & 0.832 & & 0.043 & 0.115 & 0.217 & 0.131 \\
\hline $\mathrm{MC}$ & 0.840 & 0.886 & & 0.128 & 0.232 & 0.078 & & 0.732 & 0.770 & & 0.004 & 0.070 & 0.017 \\
\hline Femur & 0.777 & 0.532 & 0.642 & & 0.144 & 0.028 & & 0.780 & 0.688 & 0.873 & & 0.315 & 0.039 \\
\hline Tibia & 0.565 & 0.524 & 0.560 & 0.580 & & 0.090 & & 0.775 & 0.679 & 0.783 & 0.628 & & 0.327 \\
\hline MT & 0.881 & 0.729 & 0.811 & 0.826 & 0.721 & & & 0.754 & 0.657 & 0.807 & 0.746 & 0.608 & \\
\hline \multicolumn{14}{|l|}{ M. lagotis } \\
\hline Humerus & & 0.085 & 0.385 & 0.038 & 0.194 & 0.138 & & & & & & & \\
\hline Radius & 0.863 & & 0.097 & 0.066 & 0.190 & 0.443 & & & & & & & \\
\hline $\mathrm{MC}$ & 0.638 & 0.730 & & 0.100 & 0.056 & 0.065 & & & & & & & \\
\hline Femur & 0.882 & 0.834 & 0.739 & & 0.030 & 0.272 & & & & & & & \\
\hline Tibia & 0.736 & 0.677 & 0.659 & 0.825 & & 0.328 & & & & & & & \\
\hline MT & 0.800 & 0.619 & 0.724 & 0.696 & 0.552 & & & & & & & & \\
\hline
\end{tabular}


Table S10: Epiphyseal partial correlation matrix for each peramelemorph species. Partial correlation values are in the lower triangle, and associated Edge exclusion values (EED) are in the upper triangle. Bold indicates significant pairwise tests (EED $\geq 3.84$ ). Grey cells are within-bone correlations and boxed cells are serially homologous limb bone correlations. MC = Metacarpal and MT= Metatarsal.

\begin{tabular}{|c|c|c|c|c|c|c|c|c|c|c|c|c|}
\hline & Humerus & Radius & MC & Femur & Tibia & MT & Humerus & Radius & MC & Femur & Tibia & MT \\
\hline I. obesulus & & & & & & & & & & & & \\
\hline Humerus & & 0.082 & 0.003 & 0.018 & 0.439 & 0.093 & & 1.216 & 1.340 & 0.512 & 0.371 & 0.010 \\
\hline Radius & 0.038 & & 4.318 & 0.029 & 0.058 & 0.964 & -0.175 & & 0.598 & 0.023 & 0.001 & 0.023 \\
\hline MC & 0.008 & 0.272 & & 0.507 & 1.621 & 7.186 & 0.184 & 0.123 & & 1.040 & 4.037 & 5.048 \\
\hline Femur & 0.018 & 0.023 & 0.095 & & 0.106 & 3.713 & -0.114 & -0.024 & 0.162 & & 0.472 & 1.255 \\
\hline Tibia & -0.088 & 0.032 & -0.169 & 0.043 & & 8.958 & -0.097 & -0.006 & 0.314 & 0.110 & & 2.784 \\
\hline MT & 0.041 & 0.131 & 0.347 & 0.253 & 0.384 & & -0.016 & 0.025 & 0.348 & -0.178 & 0.263 & \\
\hline I. macrourus & & & & & & & & & & & & \\
\hline Humerus & & 10.687 & 4.043 & 18.662 & 18.331 & 14.516 & & 1.911 & 0.014 & 9.495 & 1.542 & 0.604 \\
\hline Radius & 0.859 & & 2.087 & 8.484 & 7.418 & 5.996 & 0.384 & & 0.695 & 5.853 & 0.478 & 0.281 \\
\hline MC & 0.630 & -0.479 & & 4.963 & 2.879 & 3.518 & 0.034 & 0.237 & & 0.094 & 0.017 & 0.039 \\
\hline Femur & -0.950 & 0.809 & 0.680 & & 15.318 & 11.866 & -0.739 & 0.621 & -0.088 & & 1.878 & 0.172 \\
\hline Tibia & 0.948 & -0.777 & -0.550 & 0.923 & & 26.010 & 0.347 & -0.198 & 0.037 & 0.381 & & 6.821 \\
\hline MT & -0.915 & 0.726 & 0.596 & -0.879 & 0.980 & & 0.222 & 0.152 & 0.057 & 0.119 & 0.658 & \\
\hline \multicolumn{13}{|l|}{ M. lagotis } \\
\hline Humerus & & 0.129 & 0.744 & 0.010 & 1.114 & 0.027 & & & & & & \\
\hline Radius & -0.113 & & 9.340 & 2.055 & 1.005 & 0.028 & & & & & & \\
\hline MC & -0.268 & 0.779 & & 2.024 & 5.154 & 0.441 & & & & & & \\
\hline Femur & -0.032 & 0.431 & -0.428 & & 0.255 & 0.303 & & & & & & \\
\hline Tibia & 0.325 & -0.309 & 0.635 & 0.159 & & 0.070 & & & & & & \\
\hline MT & -0.052 & -0.053 & 0.208 & -0.173 & 0.084 & & & & & & & \\
\hline
\end{tabular}

